\title{
Managing toxicities associated with immune checkpoint inhibitors: consensus recommendations from the Society for Immunotherapy of Cancer (SITC) Toxicity Management Working Group
}

I. Puzanov ${ }^{1 \dagger}$, A. Diab ${ }^{2 \dagger}$, K. Abdallah ${ }^{3}$, C. O. Bingham III', C. Brogdon ${ }^{5}$, R. Dadu², L. Hamad ${ }^{1}$, S. Kim², M. E. Lacouture ${ }^{6}$, N. R. LeBoeuf ${ }^{7}$, D. Lenihann ${ }^{8}$, C. Onofrei ${ }^{9}$, V. Shannon², R. Sharma' ${ }^{1}$, A. W. Silk ${ }^{12}$, D. Skondra ${ }^{10}$, M. E. Suarez-Almazor ${ }^{2}$, Y. Wang ${ }^{2}$, K. Wiley ${ }^{11}$, H. L. Kaufman ${ }^{12+}$, M. S. Ernstoff ${ }^{1 *+}$ and on behalf of the Society for Immunotherapy of Cancer Toxicity Management Working Group

\begin{abstract}
Cancer immunotherapy has transformed the treatment of cancer. However, increasing use of immune-based therapies, including the widely used class of agents known as immune checkpoint inhibitors, has exposed a discrete group of immune-related adverse events (irAEs). Many of these are driven by the same immunologic mechanisms responsible for the drugs' therapeutic effects, namely blockade of inhibitory mechanisms that suppress the immune system and protect body tissues from an unconstrained acute or chronic immune response. Skin, gut, endocrine, lung and musculoskeletal irAEs are relatively common, whereas cardiovascular, hematologic, renal, neurologic and ophthalmologic irAEs occur much less frequently. The majority of irAEs are mild to moderate in severity; however, serious and occasionally life-threatening irAEs are reported in the literature, and treatment-related deaths occur in up to $2 \%$ of patients, varying by ICI. Immunotherapy-related irAEs typically have a delayed onset and prolonged duration compared to adverse events from chemotherapy, and effective management depends on early recognition and prompt intervention with immune suppression and/or immunomodulatory strategies. There is an urgent need for multidisciplinary guidance reflecting broad-based perspectives on how to recognize, report and manage organ-specific toxicities until evidence-based data are available to inform clinical decision-making. The Society for Immunotherapy of Cancer (SITC) established a multidisciplinary Toxicity Management Working Group, which met for a full-day workshop to develop recommendations to standardize management of irAEs. Here we present their consensus recommendations on managing toxicities associated with immune checkpoint inhibitor therapy.
\end{abstract}

Keywords: Immune-related adverse events, Toxicity, Immune checkpoint inhibitor

\footnotetext{
* Correspondence: Marc.Ernstoff@RoswellPark.org

${ }^{\dagger}$ Equal contributors

'Roswell Park Cancer Institute, Elm \& Carlton Streets, Buffalo, NY 14263, USA

Full list of author information is available at the end of the article
} 


\section{Background}

Cancer immunotherapy has revolutionized the treatment of cancer [1,2]. Currently, the most widely used approach is the administration of targeted monoclonal antibodies (mAbs) directed against regulatory immune checkpoint molecules that inhibit $\mathrm{T}$ cell activation [1]. At present, six immune checkpoint inhibitors (ICIs) are approved by the U.S Food and Drug Administration (FDA) for use in a variety of solid tumors, and one hematological malignancy (Hodgkin lymphoma) [3]. Ipilimumab, a fully human IgG1 mAb that blocks the cytotoxic $\mathrm{T}$ lymphocyte-antigen-4 (CTLA-4), a checkpoint inhibitor of $\mathrm{T}$ cell activation, was the first ICI approved, in 2011, for use in advanced melanoma [4]. Pembrolizumab and nivolumab, both engineered IgG4 mAbs that regulate $\mathrm{T}$ cell activation by blocking the protein programmed death 1 (PD-1), received FDA approval in patients with advanced melanoma in $2014[5,6]$ and the indications for both have subsequently expanded considerably. Indeed, in a landmark regulatory step, the FDA recently approved both pembrolizumab and nivolumab for use in certain patients with mismatch repair deficient (dMMR) and microsatellite instability high (MSI-H) cancers that have progressed following treatment with chemotherapy - the first such 'tissue-agnostic', biomarkerdriven approvals granted $[5,6]$. Both anti-PD-1 agents are associated with negligible antibody-dependent cellmediated cytotoxicity (ADCC), a process that could be detrimental to the activation of $\mathrm{T}$ effector cells. After approval of nivolumab for the treatment of non-small cell lung carcinoma (NSCLC) in 2015, the first immunotherapy combination of ipilimumab plus nivolumab was granted approval later in 2015, again in advanced melanoma [5]. More recently, the FDA approved three new ICIs, atezolizumab, durvalumab and avelumab, all of which are antibodies directed against the protein programmed death-ligand 1 (PD-L1). Both atezolizumab and durvalumab are engineered IgG1 mAbs that include Fc modifications that eliminate ADCC, while avelumab includes a wildtype IgG1 framework with intact ADCC. Since May 2016, atezolizumab and durvalumab have both been approved for the treatment of NSCLC and urothelial carcinoma, and avelumab was approved for use in Merkel cell carcinoma and urothelial carcinoma [7-9].

Immune-related adverse events (irAEs) are discrete toxicities caused by non-specific activation of the immune system, and can affect almost any organ system. In some studies, the reported incidence is as high as $90 \%$ for any-grade irAEs due to single-agent ICI therapy [10], but meta-analysis indicates an overall incidence $<75 \%$ with anti-CTLA-4 monotherapy (ipilimumab) [11], and $\leq 30 \%$ in phase 3 trials of anti-PD-1/PD-L1 agents [12-14]. IrAEs $\geq$ grade 3 severity occur in up to $43 \%$ of patients taking ipilimumab [10] and $\leq 20 \%$ taking PD-1/PD-L1 agents
$[12,15]$. The incidence of irAEs with ipilimumab and pembrolizumab is dose-dependent, with greater toxicity at higher dose levels; toxicity also varies between the adjuvant and metastatic disease settings [10, 16-19]. There is significant variance in definitions of toxicity severity across disciplines, as well as variation in how symptoms and signs that may be attributable to the same underlying pathophysiology are reported. This causes considerable difficulty in obtaining accurate data on incidence and prevalence based on clinical trials [12]. Nonetheless, the incidence of most irAEs with ICI monotherapy appears to be broadly similar across tumor types [12]. Some of the mechanisms that underpin the development of inflammatory toxicity - in particular those driven by CD8 T cell activity - overlap with those responsible for the drugs' therapeutic effects. However, the exact pathogenesis of immune toxicity is not clear, and many other inflammatory cells, such as Th17 and other types of cells, are reported to be involved. The mechanism of toxicity may also vary by ICI, and may ultimately affect acuity, chronicity and management. In some cases, irAEs may occur in patients with durable responses to treatment; this association has not been fully ascertained [20, 21].

With increasing patient exposure to immunotherapy, the nature and range of irAEs is becoming more clearly defined, and several new but serious adverse events have been reported [22]. Skin, gut, endocrine, lung and musculoskeletal irAEs are relatively common, whereas, cardiovascular, hematologic, renal, neurologic and ophthalmologic irAEs are well-recognized but occur much less frequently (Fig. 1). Although the majority of irAEs are mild to moderate in severity, serious, occasionally life-threatening irAEs (e.g., severe colitis, pneumonitis, encephalitis, toxic epidermal necrolysis, myocarditis, and autoimmune type I diabetes mellitus [T1DM] presenting as diabetic ketoacidosis), are reported in the literature, and treatment-related deaths have been reported in up to $2 \%$ of patients in clinical trials $[14,23,24]$. As life-threatening irAEs are rare, and may mimic other better-known conditions, there is growing recognition of the need to educate both the oncology and general medical communities in recognizing and instituting urgent and appropriate treatment of these conditions.

Immune-related AEs resulting from immunotherapy can have a delayed onset and prolonged duration compared to adverse events resulting from chemotherapy (Fig. 2), in part due to pharmacodynamic differences. Moreover, the relationship between irAEs and dose/exposure remains to be fully established [25]. As such, clinicians must remain vigilant to the diverse clinical presentations of irAEs and the possibility that patients may present with irAEs late in the course of treatment, and - in some cases - months or even years after treatment discontinuation [26, 27]. Nonetheless, since diagnostic tests may be invasive and potentially costly, investigations should be undertaken 

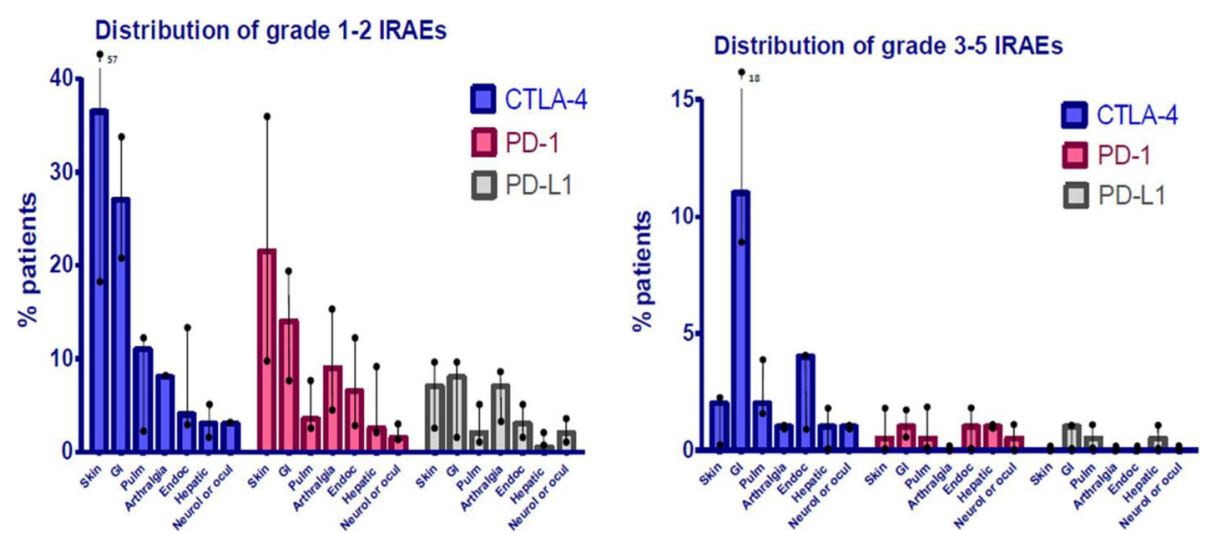

Fig. 1 Distribution of mild and severe immune-related adverse events (irAEs) associated with immune checkpoint inhibitor therapy. [Adapted from [88]]

judiciously and reserved for situations when the results will guide patient management. Table 1 provides a list of recommended tests to consider in all patients prior to initiating checkpoint inhibitor therapy.

Effective management of irAEs depends on early recognition and prompt intervention with immune suppression and/or immunomodulatory strategies appropriate to the affected organ and the severity of toxicity. Specialist physicians, nurses and pharmacists familiar with irAEs should be involved early, and hospitalization may be necessary in serious ( $\geq$ grade 4 ) or grade 3 irAEs that do not respond to therapy, or to expedite work-up and prevent complications from potentially life-threatening irAEs [28]. Patient education on the potential for irAE development is a key component of any pre-treatment discussion with patients considered suitable candidates for immunotherapy. It is also important to establish physician networks to share outcomes of successful irAE treatment strategies. Short-term adverse events due to the use of moderate to high dose corticosteroids (e.g., opportunistic infections, sleep disturbance, gastritis, and hypertension) should be anticipated. Patients receiving long-term or high dose corticosteroids are at risk of developing diabetes mellitus and osteoporosis and should receive vitamin $\mathrm{D}$ and calcium supplementation and, in some cases, antibiotic prophylaxis [28]. However, conflicting reports on the associated between antibiotic use and ICI efficacy pose as yet unanswered about whether routine antimicrobial prophylaxis is appropriate in patients receiving ICIs $[29,30]$. For steroid-refractory cases and/or when steroid sparing is desirable, management should be coordinated with disease specialists. Other immunomodulatory agents, such as infliximab, other tumor necrosis factor inhibitors (TNFi), mycophenolate mofetil, anti-thymocyte globulin (ATG), calcineurin inhibitors, methotrexate, or intravenous immunoglobulin (IVIG) and plasmapharesis may be required. However, besides TNFi for colitis, these immunosuppressive treatments have not been evaluated in large numbers of patients. Some retrospective analyses suggest that use of corticosteroids for the management of irAEs is not associated with inferior results of therapy [31, 32] but, due

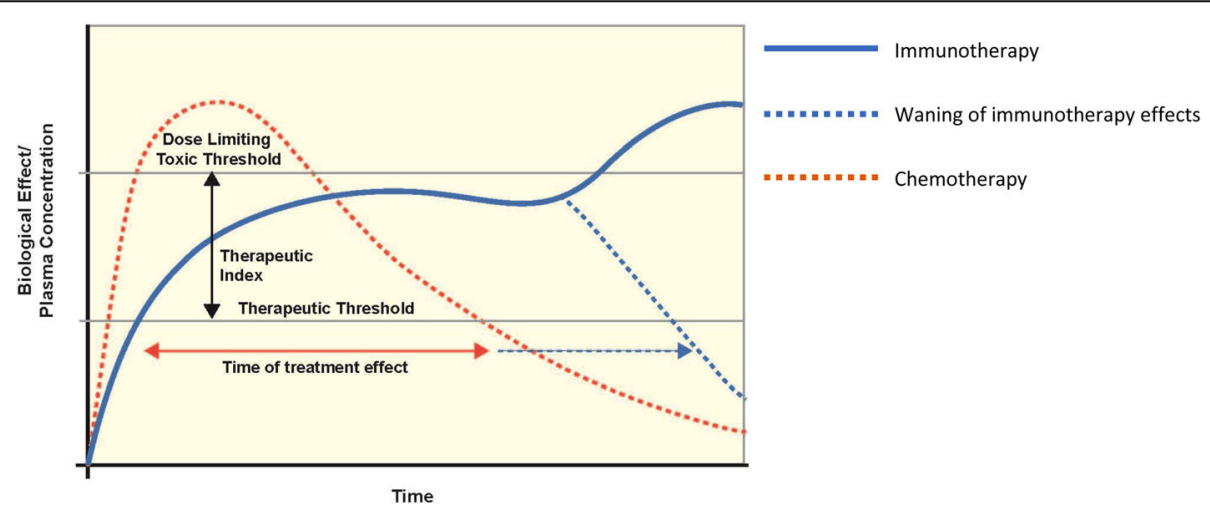

Fig. 2 Pharmacokinetic/pharmacodynamic differences between chemotherapy and immunotherapy. Reproduced with permission from [25]. Dotted blue line represents waning of the biological effects of immunotherapy over time, and solid blue line represents early or late toxic effects. Horizontal dotted blue arrow therefore represents duration of immunotherapy treatment benefit 
Table 1 Pre-treatment evaluation and diagnostic tests to consider in all patients prior to initiating checkpoint inhibitor therapy

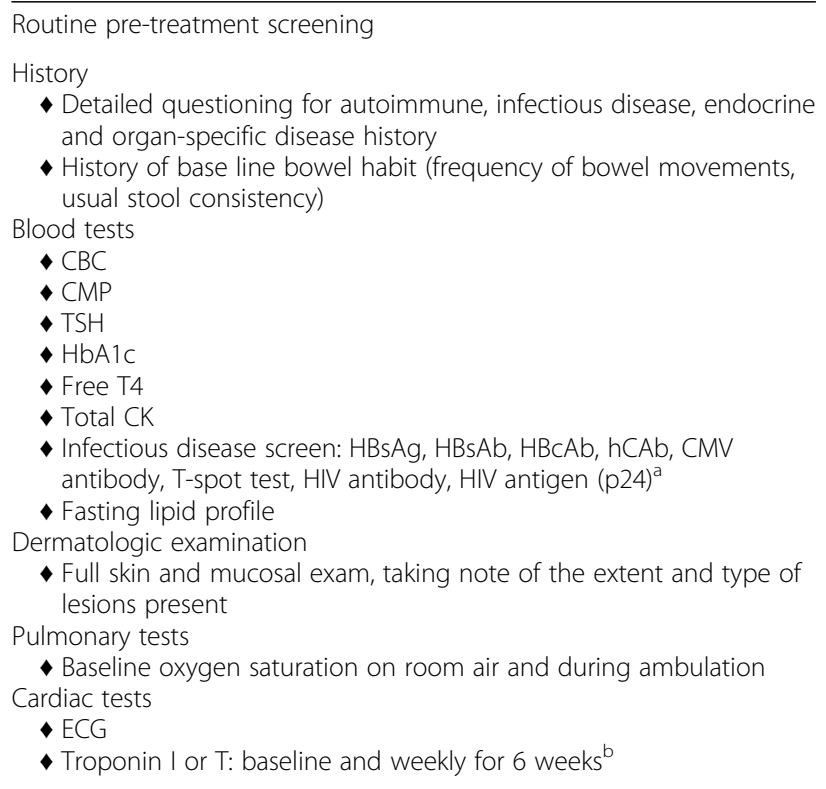

Additional screening tests recommended in patients with pre-existing organ disease/at risk of organ-specific toxicity

Endocrine tests

- 8 am cortiso

- 8 am ACTH

Cardiac tests

- Brain natriuretic peptide (BNP) or N-terminal pro B-type natriuretic peptide (NT pro-BNP)

Pulmonary tests

- PFTs ${ }^{\mathrm{C}}$

- $6 \mathrm{MWT}^{\mathrm{C}}$

In certain settings, some of these tests may not be readily available. Until their use is firmly supported by evidence, individual physician judgment is recommended ${ }^{\text {a }}$ These tests become very relevant if patients develop irAEs and require immunosuppressive treatment such as steroids and/or anti-TNFa treatment ${ }^{b}$ Given the rarity of cardiac toxicity, this may not be cost-effective as a routine test. . Baseline troponin should be measured although the follow up interval for re-testing is not determined. Any suspicious cardiopulmonary symptoms warrant repeat troponin and natriuretic testing in this population

'Given the rarity of pulmonary toxicity, pre-treatment PFTs and 6MWTs should considered in patients with pre-existing lung disease (chronic obstructive pulmonary disease, interstitial lung disease, sarcoidosis, pulmonary fibrosis etc.) and may not be feasible in all patients

ACTH, Adrenocorticotropic hormone; CBC, Complete blood count; CMP, Complete metabolic panel; CMV, Cytomegalovirus; CK, Creatine kinase; ECG, Electrocardiogram; HbA1c, Glycosylated hemoglobin; HBsAg, Hepatitis B surface antigen; $\mathrm{HBsAb}$, Hepatitis B surface antibody; $\mathrm{HBcAb}$, Hepatitis B core antibody; HCAb, Hepatitis C antibody; HIV, Human Immunodeficiency Virus; PFTs, Pulmonary function tests; TSH, Thyroid-stimulating hormone; T4, Thyroxine; $6 \mathrm{MWT}, 6$ min walk test

to confounding, the association of irAEs with immunologic activity from immunosuppression, and with individual patient efficacy, is not clear. The effects of alternative forms of immunosuppression on the efficacy of ICIs have not yet been sufficiently studied.

As physicians, nurses and patients become aware of the value of immune-based treatments, including the synergies offered by combination immunotherapy strategies, there is a pressing need for guidance on how to recognize, report and manage irAEs that arise in the course of treatment. The Common Terminology Criteria for Adverse Events (CTCAE) [33], a descriptive lexicon of terms and adverse event severity, was developed by the National Cancer Institute (NCI) at the National Institutes of Health $(\mathrm{NIH})$, with the goal of standardizing AE reporting across medical specialties. However, increasing use of immunotherapy has clarified limitations in how immune-related toxicities are addressed and classified within the current CTCAE, as well as in other databases such as the Medical Dictionary for Regulatory Activities (MedDRA). Importantly, the need for formal pathways for reporting suspected irAEs has also highlighted the tendency for CTCAE grading to under- or over-estimate true irAE incidence and/or severity [28]. In certain settings, such as with rheumatologic irAEs, CTCAE criteria are difficult to apply and do not allow accurate recording of the severity and impact of irAEs, especially as conditions may become chronic [34]. These shortcomings present an opportunity to improve and streamline irAE reporting in the next versions of CTCAE and MedDRA. Similarly, since drug labels for FDA-approved checkpoint inhibitors are based on clinical trial data for individual drugs and do not always align across therapeutic class, clinicians need multidisciplinary, broad perspective guidance on how to manage organ-specific toxicities.

To this end, the Society for Immunotherapy of Cancer (SITC) established a Toxicity Management Working Group to develop consensus recommendations on management of irAEs that develop following ICI therapy until evidencebased data are available to inform clinical decision-making. This report represents the outcome of a recent workshop to standardize toxicity management. The results represent consensus thinking by a multidisciplinary group of experts in the field but should not replace sound clinical judgment or personalized drug management, as immunotherapy patients often require highly individualized management.

\section{Methods \\ Consensus group representation}

In response to the need for a collaborative, multidisciplinary approach to the management of ICI toxicities, the SITC convened a one-day workshop on March 31st, 2017, in Washington D.C. The meeting was a multi-stakeholder effort with participation from approximately 85 experts from academia, government, industry, scientific organizations and other related entities. Representation was sought from medical oncologists, surgeons, disease subspecialists, basic scientists, pharmacists, industry clinical, regulatory and safety experts and nurses. In order to streamline recommendations across the range of organizations active in the area of cancer immunotherapy, SITC invited representatives from the American Society of Clinical 
Oncology (ASCO), National Comprehensive Cancer Network (NCCN), Parker Institute for Cancer Immunotherapy, Friends of Cancer Research, American Association for Cancer Research (AACR), Association of Community Cancer Centers (ACCC), NCI and the Oncology Nursing Society (ONS) to participate in the workshop. To ensure that commercial interests did not influence the outcomes of the workshop, industry representatives participated in group discussions but final approval of the workshop output, and of this manuscript, was the responsibility of the organizing committee, none of whom are employed by a pharmaceutical or biotechnology company. Representatives from the Office of Hematology and Oncology Products, Center for Drug Evaluation and Research (CDER), were invited to review and provide feedback on the final manuscript. Individuals selected as authors were workshop organizers and lead discussants for individual organ-specific toxicity breakout groups. All participants were required to disclose any potential conflicts of interest prior to participation.

\section{Workshop objectives and procedures}

The overarching goals of the workshop were twofold: 1) to develop treatment algorithms for managing common and rare immunotherapy-related toxicities and 2) to develop standardized templates, including inclusion and exclusion criteria, for irAE management in clinical trial protocols (which will be reported separately). More broadly, participants were charged with describing the spectrum of immune-related toxicities and providing recommendations on recognizing, monitoring and managing these toxicities. To facilitate discussion among experts in different medical fields, attendees broke out into 11 subgroups ('breakout groups') that focused on irAEs identified by body system (dermatologic, gastrointestinal, endocrine, pulmonary, rheumatologic, cardiovascular, hematologic, renal, neurologic and ophthalmologic) as well as infusion reactions. These breakout groups were generally supplemented with disease subspecialty expertise focused on the area of interest. Each breakout group received instructions to guide their discussion, a list of recognized toxicities by system, relevant drug package inserts, several key supporting references, and a copy of CTCAE version 4.0. A working draft of the Friends of Cancer Research/Parker Institute for Cancer Immunotherapy guidelines on monitoring, management and follow-up of irAEs from anti-PD-1/PD-L1 agents was also distributed [35].

After separate breakout group discussions, one representative from each group presented their recommendations to all participants, and responded to questions and additional suggestions from the wider group. Following the meeting, recommendations made on-site were recirculated by email to participants from each breakout group to ensure all views and opinions were captured. The final recommendations on management of irAEs presented in this paper therefore represent the views of each multidisciplinary expert group. These recommendations are not intended to provide comprehensive medical guidance on the management of disorders that may arise from use of immunotherapy treatment; specialist care should be sought as necessary, and as indicated in treatment-specific guidelines.

\section{Strengths and limitations of the consensus recommendations}

These consensus recommendations represent the views of a broad range of experts from multiple fields of expertise, and from large cancer organizations with differing areas of focus. In some cases they are driven by evidence from the published literature; in others, particularly where data are lacking, they are guided by accumulated clinical experience and practice. The participation of stakeholders from the pharmaceutical and biotechnology industries is another strength, ensuring that those involved in drug research and development are part of the discussion and that there is access to large industrycollected patient databases. However, it is important to acknowledge that evidence gaps are considerable, consensus was not reached on all issues, and many questions remain unanswered. Furthermore, not all working groups had representation from all specialist groups (oncologist, disease specialist, nurse, pharmacist). The recommendations may not take into account reimbursement restrictions that could limit access to recommended drugs for some patients. Lastly, but importantly, there was no patient representation. Finally, the recommendations addressed in this document reflect irAEs related to PD-1/PD-L1 and CTLA-4 inhibitors, and do not address toxicity that may ensue following administration of other classes of immunotherapy, including chimeric antigen receptor $\mathrm{T}$ cell (CAR T) therapy. It is unclear to what extent the recommendations can be generalized to immunotherapy agents other than those addressed in this manuscript, including agents in development.

\section{Consensus recommendations}

The recommendations for managing toxicities associated with ICIs, below, represent the consensus views of participants in the 11 body system groups. Overall, irAEs are broken down into two major categories, based on the opinions of the workshop organizers regarding the frequency with which they are seen in clinical practice: frequently reported (dermatologic, gastroenterological, endocrine, respiratory, and rheumatologic/musculoskeletal) and uncommon (cardiovascular, hematologic, renal, neurologic and ophthalmologic). Infusion reactions, which are more common with mAbs based on a wildtype IgG1 
backbone and less common with IgG4 antibodies, are also addressed. Within each body system, information is divided into three sections: clinical presentation and epidemiology, diagnostic evaluation, and guidance on when to refer to a disease specialist.

Management of irAEs relies heavily on corticosteroids, and other immunomodulatory agents, which should be prescribed judiciously to reduce the potential for short and long-term complications. It remains unclear whether prophylactic antibiotics should routinely be prescribed to reduce the potential for opportunistic infection in patients receiving steroids. Broadly, corticosteroid management can be approached as shown in Table 2, but treatment should be individualized depending on each patient's medical history; co-morbidities; underlying disease status; type, number and severity of adverse events; ICI administered; and ability to tolerate corticosteroids.

Table 3 summarizes the recommended management of recognized irAEs across body systems.

\section{Frequently reported immune-related adverse events Dermatologic adverse events}

Clinical presentation and epidemiology Maculopapular rash and pruritus are common reactions to ICIs but lichenoid, eczematous, and bullous dermatitis, and psoriasis have also been reported, albeit less frequently. Vitiligo is frequently seen in the melanoma patient population. Dermatologic toxicity (all grades) is reported in 30-40\% of patients taking PD-1/PD-L1 inhibitors [13, 15], and approximately $50 \%$ of patients treated with ipilimumab [13]. A systematic review of the literature reported that $13-20 \%$ of patients taking pembrolizumab or nivolumab developed rash or pruritus (all-grade) and approximately $8 \%$ (all with melanoma) developed vitiligo [36], which is associated with tumor response [20]. More recently, several cases of hair re-pigmentation have also been described in patients treated with anti-PD1 or anti-PD-L1 therapy [37]. Onset of skin irAEs typically occurs within days or weeks of treatment [38] although onset may be delayed, appearing after several months of treatment [39]. Most dermatologic irAEs are low-grade and manageable, $[13,36]$ although rare, potentially life-threatening exfoliative dermatological conditions such as Stevens-Johnson Syndrome/toxic epidermal necrolysis (SJS/TEN), and drug rash with eosinophilia and systemic symptoms (DRESS) have been reported [28]. Severe irAEs tend to occur more commonly with combination ICI therapy [40]. Any clinical suspicion of such reactions should prompt immediate specialist referral. Permanent discontinuation of immunotherapy is mandatory for grade 4 dermatologic irAEs, SJS/ TEN, or DRESS syndrome.

Diagnostic evaluation: Given the frequency and persistence of skin toxicities with ICIs, dermatologic assessments are warranted in patients with a known history of immune-related skin disorders such as psoriasis, bullous pemphigoid or lupus. Non-specific maculopapular eruptions are commonly reported, which may, in part, reflect the limitations of CTCAE in the classification of specific subsets of skin disorders. Whenever possible, the irAE should be categorized since management algorithms reflect the approach to idiopathic skin disorders, beyond systemic immune suppression with steroids. Patients

Table 2 General guidance for corticosteroid management of immune-related adverse events

\begin{tabular}{|c|c|c|}
\hline $\begin{array}{l}\text { Grade of immune-related AE } \\
\text { (CTCAE/equivalent) }\end{array}$ & Corticosteroid management & Additional notes \\
\hline 1 & - Corticosteroids not usually indicated & - Continue immunotherapy \\
\hline 2 & $\begin{array}{l}\text { - If indicated, start oral prednisone } 0.5-1 \mathrm{mg} / \mathrm{kg} / \text { day if patient } \\
\text { can take oral medication. } \\
\text { - If IV required, start methylprednisolone } 0.5-1 \mathrm{mg} / \mathrm{kg} / \text { day IV } \\
\text { - If no improvement in 2-3 days, increase corticosteroid dose to } \\
2 \mathrm{mg} / \mathrm{kg} / \text { day } \\
\text { - Once improved to } \leq \text { grade } 1 \mathrm{AE} \text {, start } 4-6 \text { week steroid taper }\end{array}$ & $\begin{array}{l}\text { - Hold immunotherapy during corticosteroid use } \\
\text { - Continue immunotherapy once resolved to sgrade } \\
1 \text { and off corticosteroids } \\
\text { - Start proton pump inhibitor for Gl prophylaxis }\end{array}$ \\
\hline 3 & $\begin{array}{l}\text { - Start prednisone } 1-2 \mathrm{mg} / \mathrm{kg} / \mathrm{day} \text { (or equivalent dose of } \\
\text { methylprednisolone) } \\
\text { - If no improvement in 2-3 days, add additional/alternative } \\
\text { immune suppressant } \\
\text { - Once improved to } \leq \text { grade 1, start 4-6-week steroid taper } \\
\text { - Provide supportive treatment as needed }\end{array}$ & $\begin{array}{l}\text { - Hold immunotherapy; if symptoms do not improve } \\
\text { in 4-6 weeks, discontinue immunotherapy } \\
\text { - Consider intravenous corticosteroids } \\
\text { - Start proton pump inhibitor for Gl prophylaxis } \\
\text { - Add PCP prophylaxis if more than } 3 \text { weeks of } \\
\text { immunosuppression expected ( }>30 \text { mg prednisone } \\
\text { or equivalent/day) }\end{array}$ \\
\hline 4 & $\begin{array}{l}\text { - Start prednisone 1-2 mg/kg/day (or equivalent dose of } \\
\text { methylprednisolone) } \\
\text { - If no improvement in 2-3 days, add additional/alternative } \\
\text { immune suppressant, e.g., infliximab } \\
\text { - Provide supportive care as needed }\end{array}$ & $\begin{array}{l}\text { - Discontinue immunotherapy } \\
\text { - Continue intravenous corticosteroids } \\
\text { - Start proton pump inhibitor for Gl prophylaxis } \\
\text { - Add PCP prophylaxis if more than } 3 \text { weeks of } \\
\text { immunosuppression expected (>30 mg prednisone } \\
\text { or equivalent/day) }\end{array}$ \\
\hline
\end{tabular}


Table 3 Recommended management of CTCAE-based immune-related adverse events due to immune checkpoint inhibitor (ICI) therapy DERMATOLOGIC Specialist referral?

Maculopapular rash/dermatitis

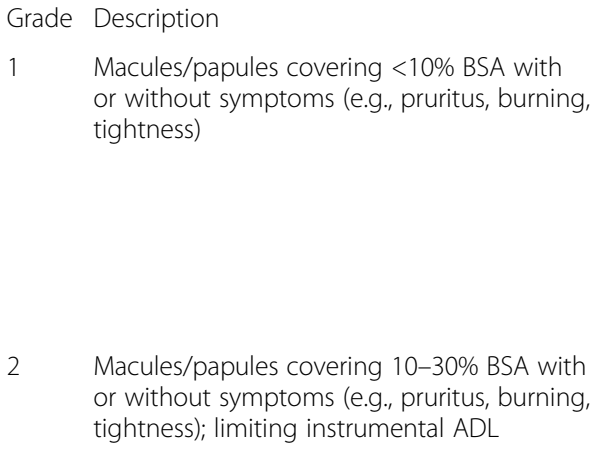

2 Macules/papules covering 10-30\% BSA with or without symptoms (e.g., pruritus, burning, tightness); limiting instrumental ADL

3 Macules/papules covering $>30 \%$ BSA with or without associated symptoms; limiting self-care ADL

Management

- Continue ICI

- Oral antihistamines

- Cetirizine/loratidine $10 \mathrm{mg}$ daily (non-sedating); hydroxyzine 10-25 mg QID, or at bedtime

- Topical corticosteroids

- Class I topical corticosteroid (clobetasol propionate, halobetasol propionate, betamethasone dipropionate cream or ointment) for body; Class VMI corticosteroid (aclometasone, desonide, hydrocortisone $2.5 \%$ cream) for face

- Continue ICI

- Non-urgent dermatology referral

- Oral antihistamines

- Cetirizine/loratidine $10 \mathrm{mg}$ daily (non-sedating); hydroxyzine 10-25 mg QID, or at bedtime

- Topical corticosteroids (see grade 1)

- As above

- Cetirizine/loratidine $10 \mathrm{mg}$ daily (non-sedating); hydroxyzine 10-25 mg QID, or at bedtime

- Hold ICl

- Same day dermatology consult

- Rule out systemic hypersensitivity: CBC with differential, CMP

- Oral antihistamines

o Cetirizine/loratidine $10 \mathrm{mg}$ daily (non-sedating); hydroxyzine 10-25 mg QID, or at bedtime

- Systemic corticosteroids

- Prednisone 0.5 - $1 \mathrm{mg} / \mathrm{kg} /$ day (or equivalent dose of methylprednisolone) until rash resolves to $\leq$ grade 1

Pruritus*

Grade Description

1 Mild or localized; topical intervention indicated

\section{Management}

- Emollients with cream or ointment based, fragrance-free products

- Class I topical corticosteroid (clobetasol propionate, halobetasol propionate, betamethasone dipropionate) for body; Class VNI corticosteroid (aclometasone, desonide, hydrocortisone $2.5 \%$ ) for face, AND oral antihistamines (e.g., cetirizine/loratidine $10 \mathrm{mg}$ daily, hydroxyzine 10$25 \mathrm{mg}$ QID, or at bedtime

2 Intense or widespread; intermittent; skin

- Dermatology referral

changes from scratching (e.g., edema, papulation, • Class I topical steroid (clobetasol propionate, halobetasol excoriation, lichenification, oozing/crusts); oral intervention indicated; limiting instrumental ADL

Intense or widespread; constant; limiting self-care ADL or sleep; oral corticosteroid or immunosuppressive therapy indicated propionate, betamethasone dipropionate) for body: class VNI steroid (aclometasone, desonide, hydrocortisone 2.5\%) for face, AND oral antihistamines (e.g., cetirizine/loratidine $10 \mathrm{mg}$ daily, hydroxyzine 10-25 mg QID, or at bedtime

- Oral corticosteroids

- Prednisone 0.5 - $1 \mathrm{mg} / \mathrm{kg} /$ day (or equivalent of methylprednisolone) tapered over 2 weeks

- Dermatology referral

- GABA agonist (pregabalin, gabapentin 100-300 mg TID)

- Oral corticosteroid

- Prednisone 0.5 - $1 \mathrm{mg} / \mathrm{kg} /$ day (or equivalent of methylprednisolone) tapered over 2 weeks

Notes:

1. Grade 4 maculopapular rash/dermatitis is not included in CTCAE

* Recommendations provided are based on case reports, series and expert consensus. Use of suggested therapies must be discussed with medical oncology based on individual patient considerations. The impact of these therapies on the anti-tumor immune response and efficacy of cancer treatment is unknown and requires further research.

Colitis 
Table 3 Recommended management of CTCAE-based immune-related adverse events due to immune checkpoint inhibitor (ICI) therapy (Continued)

Asymptomatic; clinical or diagnostic
observations only; intervention not indicated
[Grade 1 diarrhea frequency $\leq 4 /$ day]

$2 \quad$ Abdominal pain; mucus or blood in stool [Grade 2 diarrhea frequency 4-6/day]
3 and Grade 3: Severe abdominal pain; change in

4 bowel habits; medical intervention indicated; peritoneal signs

[Grade 3 diarrhea frequency $\geq 7 \times /$ day]

Grade 4: Life-threatening consequences; urgent intervention indicated
- Close follow up within 24-48 h for changes or progression

- Continue ICI

- If symptoms persist, start routine stool and blood tests

- Bland diet advisable during period of acute diarrhea

- Anti-diarrheal medication is optional but not highly recommended when infectious work-up is negative.

- Hold ICl

- Outpatient stool and blood work; CRP, ESR, fecal calprotectin, lactoferrin, imaging and endoscopy are optional

- If diarrhea only, observe for 2-3 days. If no improvement start prednisone $1 \mathrm{mg} / \mathrm{kg} /$ day (or equivalent dose of methylprednisolone); anti-diarrheal medication is not recommended

- If diarrhea and colitis symptoms (abdominal pain +/- blood in $\mathrm{BM}$ ), start prednisone $1 \mathrm{mg} / \mathrm{kg} /$ day (or equivalent dose of methylprednisolone)immediately

o If no improvement in $48 \mathrm{~h}$, increase corticosteroid dose to prednisone $2 \mathrm{mg} / \mathrm{kg} /$ day (or equivalent dose of methylprednisolone)

- If patient improves

- Taper corticosteroid over 4-6 weeks may be needed

- Resume ICI when corticosteroid is tapered to $\leq 10 \mathrm{mg} /$

day and patient remains symptom-free (grade $\leq 1)^{*}$

- Continue anti-PD-1 or anti-PD-L1 monotherapy

- If using combination anti-CTLA-4/anti-PD-1

immunotherapy, continue anti-PD-1 agent only

- $\mid \mathrm{Cl}$ dose reduction is not recommended

- If colitis returns on resuming $\mathrm{ICl}$ :

$\circ$ Grade $\leq$ 2: temporarily hold $\mathrm{ICl}$

- Grade $\geq 3$ : permanently discontinue $\mid \mathrm{Cl}$

- Grade 3: withhold ICl; consider resuming $\mathrm{ICI}$ when corticosteroid is tapered to $\leq 10 \mathrm{mg} /$ day and patient remains symptom-free (grade $\leq 1)$. Consider hospitalization

- Grade 4: permanently discontinue $\mathrm{ICl}$ and hospitalize

- Blood and stool infection work-up, inflammatory markers, imaging, endoscopy and $\mathrm{Gl}$ consult

- Start intravenous prednisone $1-2 \mathrm{mg} / \mathrm{kg} /$ day (or equivalent dose of methylprednisolone) immediately

- If patient improves, follow instructions for 'If patient improves' for grade 2

- If refractory or no improvement on IV corticosteroid, start prednisone $2 \mathrm{mg} / \mathrm{kg} /$ day (or equivalent dose of methylprednisolone) for 3 days

- Consider other anti-inflammatory agents e.g. infliximab 5 mg/ $\mathrm{kg}$, which can be given again after two weeks if a second dose is needed. Vedolizumab may also be used (see Note 4 below).

Notes:

1. CBC with differential, CMP, ESR and CRP are recommended before starting immunotherapy, to provide baseline values for comparison over time. Despite the association between elevated ESR and CRP and colitis, some insurance companies may not cover these tests.

2. There is no proven role for prophylactic corticosteroids (budesonide) to prevent Gl irAEs [45, 47].

3. Response to infliximab generally occurs within 1-3 days although some patients benefit from a second dose after 2 weeks. Prolonged oral prednisone taper may be required after infliximab administration. Whether infliximab reduces the antitumor efficacy of ipilimumab remains unknown [103].

4. Case reports of successful treatment of steroid-dependent immune-related colitis using vedolizumab indicate this may benefit certain patients.

5. A Gl consult is warranted in any patient who meets criteria for grade 2 diarrhea/colitis with negative infectious stool work up.

Hepatitis

Grade CTCAE Description (Note 1)

$1 \quad$ AST, ALT > ULN $-3 \times U L N$; total bilirubin > ULN-1.5XULN
Management

- Continue IC

- CMP or hepatic function panel once weekly

- If liver enzyme and function tests are stable, reduce frequency of blood tests

- Hold ICI

- Rule out viral hepatitis, autoimmune disease, biliary obstruction, new metastasis or thrombosis

\section{See note 5}


Table 3 Recommended management of CTCAE-based immune-related adverse events due to immune checkpoint inhibitor (ICI) therapy (Continued)

- Start prednisone $0.5-1 \mathrm{mg} / \mathrm{kg} /$ day (or equivalent dose of

methylprednisolone) with 4 week taper

- Monitor CMP twice a week

- Liver biopsy is optional

- Resume $\mathrm{ICl}$ when corticosteroid taper to $10 \mathrm{mg} /$ day (toxicity grade $\leq 1)$

3 and AST, ALT $>5 x U L N$; total bilirubin $>3 x U L N$
- Permanently discontinue ICl

- Monitor CMP every 1-2 days

- Start prednisone 1-2 mg/kg/day

- If refractory after 3 days, consider mycophenolate

- If liver enzymes improve, taper corticosteroid over 4 weeks

- Consider liver biopsy

Notes:

1. Liver enzyme levels stated here are not defined in CTCAE and are instead drawn from reference [104]

2. In patients with liver metastasis, ICl can be used at baseline liver profile equivalent to grade 2 . If $\geq 50 \%$ elevation in AST/

ALT lasting for $\geq 1$ week, permanently stop $\mathrm{ICl}$.

ENDOCRINE

Specialist referral?

Hypophysitis

Grade CTCAE Description*

Asymptomatic or mild symptoms; clinical or diagnostic observations only; intervention not indicated

2 Moderate; minimal, local or noninvasive intervention indicated; limiting ageappropriate instrumental ADL

3 Severe or medically significant but not immediately life-threatening; hospitalization or prolongation of existing hospitalization indicated; disabling; limiting self-care ADL

$4 \quad$ Life-threatening consequences; urgent intervention indicated
Management

- Hold ICl if $\geq$ grade 2 irAE until work up is completed and appropriate hormone replacement is started

- If central adrenal insufficiency: start physiologic steroid replacement: Hydrocortisone $\sim 10 \mathrm{mg} / \mathrm{m}^{2}$ (HC $15 \mathrm{mg}$ am, $5 \mathrm{mg}$ at $3 \mathrm{pm}$ ) o Periodic assessment (e.g., every 3 months in the first year, every 6 months thereafter): clinical monitoring and repeat hormone levels (am cortisol and ACTH and/or low dose cosyntropin stimulation test) to assess recovery

- If central hypothyroidism: start thyroid hormone (levothyroxine $1 \mathrm{mcg} / \mathrm{kg}$ )

o Repeat thyroid function testing 6-8 weeks after initiation of thyroid hormone and then periodically (e.g., every

3 months in the first year and every 6 months thereafter) to assess recovery

- If central hypogonadism, repeat hormone levels in 2-3 months and consider testosterone in men or HRT in women if appropriate for cancer type

For severe/life-threatening symptoms such as adrenal crisis, severe headache, visual field deficiency:

- Hospitalize as appropriate.

- High dose corticosteroid (prednisone $1 \mathrm{mg} / \mathrm{kg} /$ day) (or equivalent dose of methylprednisolone) in the acute phase, followed by taper over 1 month.

- Adrenal crisis should be managed per standard guidelines.

- If central hypothyroidism, replace thyroid hormone (see above) after corticosteroids have been initiated

Note: In the uncommon scenario of MRI findings without pituitary deficiency, consider high dose corticosteroids for prevention of hormonal dysfunction.

* Hypophysitis is not defined in CTCAE Version 4.0. This classification is drawn from the CTCAE category 'Endocrine disorders - Other'.

Hypothyroidism

Grade CTCAE Description

$1 \quad$ Asymptomatic; clinical or diagnostic observations only; intervention not indicated

2 Symptomatic; thyroid replacement indicated; limiting instrumental ADL

3 Severe symptoms; limiting self-care ADL; hospitalization indicated

$4 \quad$ Life-threatening consequences; urgent intervention indicated
Management

- Hold ICI for $\geq$ grade 3 irAEs

- $\mathrm{ICl}$ can be continued after resolution of symptoms to grade 2 or better.

- Start standard thyroid replacement therapy: initial dose can be the full dose $(1.6 \mathrm{mcg} / \mathrm{kg})$ in young, healthy patients, but a reduced dose of $25-50 \mathrm{mcg}$ should be initiated in elderly patients with known cardiovascular disease.

- Repeat TSH and free T4 testing after 6-8 weeks and adjust thyroid hormone dose accordingly. If TSH is above reference range, increase thyroid hormone dose by $12.5 \mathrm{mcg}$ to $25 \mathrm{mcg}$

- After identification of the appropriate maintenance dose, further evaluation is required every year, or sooner if patient's status changes 
Table 3 Recommended management of CTCAE-based immune-related adverse events due to immune checkpoint inhibitor (ICI) therapy (Continued)

- After identification of the appropriate maintenance dose, further evaluation is required every year, or sooner if patient's status changes

Hyperthyroidism

\begin{tabular}{|c|c|c|}
\hline rade & CTCAE Description & Management \\
\hline & $\begin{array}{l}\text { Asymptomatic; clinical or diagnostic } \\
\text { observations only; intervention not indicated }\end{array}$ & \multirow{4}{*}{$\begin{array}{l}\text { - Hold ICI for } \geq \text { grade } 3 \text { irAEs } \\
\text { - Standard therapy for hyperthyroidism should be followed } \\
\text { - Thyroiditis is self-limiting and has } 2 \text { phases: } \\
\text { o In the hyperthyroid phase, patients may benefit from } \\
\text { beta blockers if symptomatic (e.g., atenolol } 25-50 \mathrm{mg} \\
\text { daily, titrate for HR }<90 \text { if BP allows). Monitor closely with } \\
\text { regular symptom evaluation and free T4 testing every } \\
2 \text { weeks. } \\
\text { o Introduce thyroid hormones (see hypothyroidism } \\
\text { management) if the patient becomes hypothyroid (low } \\
\text { free T4/T3, even if TSH is not elevated). } \\
\text { - Graves' disease should be treated per standard guidelines. }\end{array}$} \\
\hline & $\begin{array}{l}\text { Symptomatic; thyroid suppression therapy } \\
\text { indicated; limiting instrumental ADL }\end{array}$ & \\
\hline & $\begin{array}{l}\text { Severe symptoms; limiting self-care ADL; } \\
\text { hospitalization indicated }\end{array}$ & \\
\hline & $\begin{array}{l}\text { Life-threatening consequences; urgent } \\
\text { intervention indicated }\end{array}$ & \\
\hline
\end{tabular}

Note: High dose corticosteroids ( $1 \mathrm{mg} / \mathrm{kg} /$ day) are not routinely required.

Type 1 diabetes (CTCAE defines hyperglycemia not diabetes)

Grade CTCAE Description

$1 \quad$ Fasting glucose $>$ ULN $-160 \mathrm{mg} / \mathrm{dL}$ (>ULN - $8.9 \mathrm{mmol} / \mathrm{L}$ )

2 Fasting glucose $>160-250 \mathrm{mg} / \mathrm{dL}$ (>8.9-13.9 mmol/L)

3 Fasting glucose $>250-500 \mathrm{mg} / \mathrm{dL}$ (>13.9-27.8 mmol/L); hospitalization indicated

$4 \quad$ Fasting glucose $>500 \mathrm{mg} / \mathrm{dL}$ (>27.8 $\mathrm{mmol} / \mathrm{L})$; life-threatening consequences

PULMONARY

Pneumonitis

\section{Grade CTCAE Description}

1 Asymptomatic; clinical or diagnostic observations only

2 Symptomatic; limiting instrumental ADL medical intervention indicated
Management

- Type 1 DM with diabetic ketoacidosis: Hold ICl; hospitalize and initiate treatment per standard guidelines.

- Type 1 DM without diabetic ketoacidosis: Hold ICI for hyperglycemia $\geq$ grade 3 . Treat with insulin and continue IC when patient recovers to grade 1.

- Treat with insulin per standard guidelines and restart ICI when patient recovers to grade 1.

- Provide patient education on diet and lifestyle modification, and blood glucose testing

Specialist referral?

Management

- Consider holding ICI

- Consider pulmonary and infectious disease consultations

- Reimage at least prior to every cycle of ICI treatment (at least every 3 weeks)

o If repeat imaging shows resolution of radiographic findings, no further CT imaging is necessary; resume therapy with close follow-up

- If evidence of progression, treat at higher grade

- If no change, consider continued therapy with close follow-up for new symptoms

- If symptoms develop, treat at higher grade

- Self-monitor symptoms and oxygen saturation (using personal pulse oximeter) every 2-3 days; weekly clinic visits

- If chest imaging abnormalities resolve, consider resuming treatment with close follow-up

- Hold ICl

- Consider hospitalization

- Pulmonary consultation for bronchoscopy with

bronchoalveolar lavage. Consider biopsies for atypical lesions

- Initiate methylprednisolone $1 \mathrm{mg} / \mathrm{kg} /$ day (IV or oral equivalent) - Day 2-3 of corticosteroids/supportive care: If symptoms improve to $\leq$ grade 2, start slow steroid taper over $>1$ month. If symptoms do not improve, or worsen, treat as grade 3-4

- Consider drug re-challenge if symptoms and imaging abnormalities resolve

- Permanently discontinue ICI

- Hospitalize; consider ICU care 
Table 3 Recommended management of CTCAE-based immune-related adverse events due to immune checkpoint inhibitor (ICI) therapy (Continued)

\begin{tabular}{lll}
\hline Life-threatening respiratory compromise; & • Pulmonary consultation for bronchoscopy with & (pulmonary and \\
urgent intervention indicated (e.g., intubation) & bronchoalveolar lavage. Consider biopsies for atypical lesions & infectious disease) \\
- Initiate methylprednisolone IV, $2 \mathrm{mg} / \mathrm{kg} / \mathrm{day}$ & - Day $2-3$ of corticosteroids/supportive care: \\
o If no clinical improvement, add infliximab or \\
cyclophosphamide, mycophenolate mofetil or IVIG \\
o If clinical improvement: reduce corticosteroids to $1 \mathrm{mg} /$ \\
kg/day and slowly taper over $>2$ months. \\
• Drug re-challenge: \\
o Grade 3: Consider drug re-challenge on a case-by-case \\
basis after discussions weighing risk/benefit with the \\
patient and only if symptoms and imaging abnormalities \\
resolve \\
o Grade 4: Permanent y discontinue ICl
\end{tabular}

Notes:

1. Consider prophylactic antibiotics for pneumocystis pneumonia (PCP) for patients receiving at least 20 mg methylprednisolone or equivalent for $\geq 4$ weeks

2. Consider calcium and vitamin D supplementation with prolonged steroid use

3. All patients with grade $2-4$ pneumonitis receiving steroids should also be on proton pump inhibitor therapy for Gl prophylaxis

4. T-spot testing should be undertaken to exclude tuberculosis in any patient being considered for anti-TNF therapy, prior to starting anti-TNF treatment.

Sarcoidosis

\section{Grade CTCAE Description}

$1 \quad$ Not defined in CTCAE

$\geq 2$

\section{Management}

- Consider holding ICI

- Close follow-up

- Consider corticosteroids

- Hold ICI

- Consider corticosteroid therapy for patients with sarcoidosis grade 2 or higher and any of the following:

o progressive radiographic change

- persistent and/or troublesome pulmonary symptoms

- lung function deterioration: TLC decline of $\geq 10 \%$, FVC decline of $\geq 15 \%$; DLCO decline of $\geq 20 \%$

o concomitant involvement of critical extrapulmonary organ systems

- sarcoid-related hypercalcemia

- Corticosteroid dose: prednisone $1 \mathrm{mg} / \mathrm{kg}$ (or IV equivalent of methylprednisolone) for grade 2 sarcoidosis or severe cases requiring hospitalization. Taper steroids over $2-4$ months, depending on response

Notes: To date, there are no studies focusing on management of sarcoidosis as a side effect of checkpoint inhibitor therapy. Current recommendations are based on clinical experience and case report publications.

RHEUMATOLOGIC/MUSCULOSKELETAL [78]

Inflammatory arthritis

\section{Grade CTCAE Description (Note 1)}

Mild pain with inflammatory symptoms (Note 2), erythema, or joint swelling (Note 3)
Management

- Continue IC

- Analgesics: NSAIDs: naproxen 500 mg BID or meloxicam 7.515 mg daily orally for 4-6 weeks

- If NSAIDs ineffective, consider prednisone 10-20 mg daily for 2-4 weeks

- Consider intra-articular corticosteroid injection only if $\leq 2$ joints affected and low dose prednisone (10 mg/day) and NSAIDs not effective

- If no improvement in 2-4 weeks, escalate to grade 2 management

- Conduct serial rheumatologic examinations (2 weeks, 4 weeks, then 4-6 weekly) and functional assessment at follow-up

- Consider holding ICI

- Rheumatology referral to confirm inflammatory arthritis, assess need for intra-articular injection and examine for signs of early bone damage 
Table 3 Recommended management of CTCAE-based immune-related adverse events due to immune checkpoint inhibitor (ICI) therapy (Continued)

- Prednisone $20 \mathrm{mg}$ daily for $2-4$ weeks, increase to $1 \mathrm{mg} / \mathrm{kg} /$ day, or equivalent. If no response in 2-4 weeks. Escalate to grade 3 management

- If symptoms improve, taper corticosteroid over 4-8 weeks or until grade 1

3 Severe pain associated with signs of inflammation, erythema, or joint swelling; irreversible joint damage (e.g., erosion); disabling; limiting self-care ADL
- Hold ICI

- Rheumatology referral

- Prednisone $1 \mathrm{mg} / \mathrm{kg} /$ day or equivalent for 2-4 weeks, or until symptoms improve to grade 1

- Consider additional immunosuppression (Note 4) (e.g. methotrexate [Note 5], sulfasalazine, leflunomide). Consider anti-cytokine therapy (e.g. TNF-inhibition) [Note 6]

- If symptoms improve, taper corticosteroid over 4-8 weeks/until grade 1; if symptoms do not improve in 4-6 weeks: permanently discontinue $\mathrm{ICl}$

Notes:

1. CTCAE includes separate listings for arthritis, joint effusion and arthralgia although there is overlap in presenting symptoms such as pain and effects on $\mathrm{ADL}$

2. Joint stiffness after sleep or inactivity, improvement of symptoms with movement or heat.

3. Joint swelling refers to the clinical finding on examination, and may encompass soft tissue swelling, joint effusion or synovitis.

4. Before initiation of these drugs, screening for hepatitis B and C should be performed

5. Methotrexate should be administered at a starting dose of $15 \mathrm{mg}$ weekly, with daily folic acid supplementation. Titrate up to a maximum of

$25 \mathrm{mg}$ weekly, or switch to injectable methotrexate if patient cannot tolerate orally

6. Before anti-cytokine therapy, evaluation for latent/active TB should be performed

INFUSION REACTIONS

Grade CTCAE Description

1 Mild transient reaction; infusion interruption not indicated; intervention not indicated

2 Therapy or infusion interruption indicated but responds promptly to symptomatic treatment (e.g., antihistamines, NSAIDS, narcotics, IV fluids); prophylactic medications indicated for $\leq 24 \mathrm{~h}$.

3 Prolonged (e.g., not rapidly responsive to symptomatic medication and/or brief interruption of infusion); recurrence of symptoms following initial improvement; hospitalization indicated for clinical sequelae

$4 \quad$ Life-threatening consequences; urgent intervention indicated

CARDIOVASCULAR

Grade CTCAE Description

1 Abnormal cardiac biomarker testing including abnormal ECG

2 Abnormal screening tests with mild symptoms

3 Moderately abnormal testing or symptoms with mild activity

Specialist referral?

Management

- Drug infusion rate may be decreased, or infusion temporarily interrupted, until resolution of the event

- Consider reducing the rate of infusion upon re-initiation or subsequent infusions

- Non-steroidal anti-inflammatory drugs (NSAIDs, e.g. acetaminophen), antihistamines, opioids, and corticosteroids may be used per investigator/ institutional guidelines

- Consider premedication for subsequent infusions per investigator/ institutional guidelines

- Permanently discontinue ICI

- For severe/life-threatening reactions, manage the patient as clinically appropriate (e.g. antihistamines, oxygen, fluids, opioids, corticosteroids, bronchodilators, etc.) per investigator/ institutional guidelines

$\checkmark$ Refer to allergist to prevent potential future reactions

Specialist referral?

Management

- Recommend baseline ECG and cardiac biomarker assessment (BNP, troponin) to establish if there is a notable change during therapy

- Mild abnormalities should be observed closely during therapy

- Control cardiac diseases (e.g. heart failure, atrial fibrillation) optimally

- Control cardiac disease risk factors proactively (including hypertension, hyperlipidemia, discontinue smoking, and monitor diabetes)

- BNP > $500 \mathrm{pg} / \mathrm{ml}$, troponin >99\% institutional normal, new ECG findings (QTc prolongation, new conduction disease, or ST-T wave changes)

- Consider withholding $\mathrm{ICl}$

o If a period of stabilization is achieved and definite cardiac toxicity was not identified, it may be reasonable to consider re-challenging the patient with $\mathrm{ICl}$, with heightened monitoring. 
Table 3 Recommended management of CTCAE-based immune-related adverse events due to immune checkpoint inhibitor (ICI) therapy (Continued)

- If confirmed cardiac injury or decompensation, hold ICI therapy

until stabilized.

- Optimally treat identified cardiac conditions

- Consider corticosteroids if myocarditis suspected (Note 2)

$4 \quad$ Moderate to severe decompensation, intravenous medication or intervention required, life threatening conditions

- Permanently discontinue $\mathrm{ICl}$

- If myocarditis is identified, consider high-dose corticosteroids (1 mg/kg methylprednisolone (IV) for at least several days) until improved to grade $\leq 1$, after that consider at least 4-5 weeks of tapering doses (Note 2).

- Add additional immunosuppressive agents in severe refractory cases.

- Give additional supportive treatments, including appropriate treatment of heart failure. Additional treatment of detected cardiac conditions should be provided.*

Notes:

1. Grades outlined here are not drawn from CTCAE.

2. Patients with confirmed myocarditis (or in cases of reasonable suspicion) should receive emergent high-dose corticosteroids. Until data are available (e.g., cut-off levels of troponin) to determine when to start corticosteroids in patients with possible (as opposed to confirmed) myocarditis, this decision should be made on a case by case basis. The importance of active, ongoing consultation with a cardiologist to discuss the risk/benefit of continuing $\mathrm{ICl}$ therapy, starting corticosteroids, or instituting other cardiac treatments, cannot be overstated.

* Other therapies for management of myocarditis or pericarditis (viral based therapy, immunoglobulins, or plasmapheresis) are speculative at this point in time.

HEMATOLOGIC

Specialist referral?

Anemia

Grade CTCAE Description

$1 \quad \mathrm{Hgb}<\mathrm{LLN}-10.0 \mathrm{~g} / \mathrm{dL} ;<\mathrm{LLN}-6.2 \mathrm{mmol} / \mathrm{L}$; $<L L N-100 \mathrm{~g} / \mathrm{L}$

$2 \quad \mathrm{Hgb}<10.0-8.0 \mathrm{~g} / \mathrm{dL} ;<6.2-4.9 \mathrm{mmol} / \mathrm{L}$; $<100-80 \mathrm{~g} / \mathrm{L}$

$3 \quad \mathrm{Hgb}<8.0 \mathrm{~g} / \mathrm{dL} ;<4.9 \mathrm{mmol} / \mathrm{L}_{;}<80 \mathrm{~g} / \mathrm{L}_{;}$ transfusion indicated

$4 \quad$ Life-threatening consequences; urgent intervention indicated

Notes:

1. No firm recommendations for corticosteroid management are provided here as treatment should be individualized.

2. If unexplained anemia does not respond to steroids, consider bone marrow biopsy.

Thrombocytopenia (CTCAE defines decreased platelet count not thrombocytopenia)

$$
\begin{array}{ll}
\text { Grade } & \text { CTCAE Description } \\
1 & <L L N-75,000 / \mathrm{mm} 3 ;<L L N-75.0 \times 10 \mathrm{e} 9 / \mathrm{L} \\
2 & <75,000-50,000 / \mathrm{mm} 3 ;<75.0-50.0 \times 10 \mathrm{e} 9 / \mathrm{L} \\
3 & <50,000-25,000 / \mathrm{mm} 3 ;<50.0-25.0 \times 10 \mathrm{e} 9 / \mathrm{L} \\
4 & <25,000 / \mathrm{mm} 3 ;<25.0 \times 10 \mathrm{e} 9 / \mathrm{L}
\end{array}
$$

\section{Management}

- Monitor closely while continuing ICI

- Monitor closely while continuing ICl

- Evaluate for possible causes and refer to hematology if no obvious cause if identified

- Hold ICI

- Consider Coombs testing and evaluation for hemolytic anemia

- Consider re-treating with ICI if hemolytic anemia responds promptly (within a few days) to corticosteroids

- Permanently discontinue ICI

\section{Management}

- Progressive or grade 3 unexplained thrombocytopenia: consider work up for autoimmune disease and rule out DIC or other cause of thrombocytopenia that may be related to underlying disease

- Precipitous development of thrombocytopenia: consider steroid intervention pending clinical condition (brain metastases, colitis, etc.) and evaluate for immune-mediated thrombocytopenia

- Permanently discontinue ICI for clinically significant, steroidrefractory $\mathrm{ICl}$-associated thrombocytopenia

Note: No firm recommendations for corticosteroid management are provided here as treatment should be individualized.

RENAL

\section{$\checkmark$}

if no cause identified$$
\checkmark
$$

$\checkmark$

if no cause identified

$\checkmark$

$\checkmark$

Nephritis

\section{Grade CTCAE Description}

1 Creatinine level increase of $>0.3 \mathrm{mg} / \mathrm{dL}$; creatinine 1.5-2.0x above baseline

\section{Management}

- Continue ICI but initiate work-up to evaluate possible causes and monitor closely 
Table 3 Recommended management of CTCAE-based immune-related adverse events due to immune checkpoint inhibitor (ICI) therapy (Continued)

\begin{tabular}{|c|c|c|c|}
\hline 2 & Creatinine $2-3 \times$ above baseline & $\begin{array}{l}\text { - Hold ICl } \\
\quad \text { o Resume when creatinine decreased to } \leq \text { grade } 1 \text { (Note 2) } \\
\text { - Consider timing of event and response to treatment when } \\
\text { making a decision } \\
\text { - Start corticosteroids (Note } 3 \text { ) } \\
\text { - Discontinue ICl for persistent or recurrent elevation }\end{array}$ & \\
\hline 3 & $\begin{array}{l}\text { Creatinine }>3 \times \text { baseline or }>4.0 \mathrm{mg} / \mathrm{dL} \text {; } \\
\text { hospitalization indicated }\end{array}$ & $\begin{array}{l}\text { - Hold ICI } \\
\text { - Consider resuming treatment if grade } 3 \text { resolves (Note 2) and } \\
\text { cause of event is confirmed. Timing of event and response to } \\
\text { treatment should be considered in making a decision } \\
\text { - Start corticosteroids (Note } 3 \text { ) } \\
\text { - Discontinue ICI for persistent or recurrent elevation }\end{array}$ & \\
\hline 4 & $\begin{array}{l}\text { Life-threatening consequences; dialysis } \\
\text { indicated }\end{array}$ & $\begin{array}{l}\text { - Permanently discontinue } \mathrm{ICl} \\
\text { - Start corticosteroids (Note 3) }\end{array}$ & $\checkmark$ \\
\hline \multicolumn{4}{|c|}{$\begin{array}{l}\text { Notes: } \\
\text { 1. Grades are those listed under 'acute kidney injury' in CTCAE [33]. } \\
\text { 2. Consider using increase from baseline rather than absolute value for creatinine monitoring, especially in patients with primary renal carcinoma or } \\
\text { other baseline renal conditions. } \\
\text { 3. For persistent creatinine elevation } \geq \text { grade } 2 \text { with no other identifiable cause, start corticosteroids. Dose and schedule should be individualized } \\
\text { and based on grade. Taper corticosteroids when creatinine improves to grade } 1 \text {. }\end{array}$} \\
\hline NEURO & LOGIC & & Specialist referral? \\
\hline \multicolumn{4}{|c|}{ Encephalopathy/Leukoencephalopathy/Reversible posterior leukoencephalopathy syndrome (PRES) } \\
\hline Grade & CTCAE Description & Management & \\
\hline 1 & Mild symptoms & $\begin{array}{l}\text { - Hold ICl and initiate diagnostic work-up } \\
\text { - Consider permanent discontinuation of ICI if AE worsens or } \\
\text { does not improve }\end{array}$ & \\
\hline 2 & Moderate symptoms; limiting instrumental ADL & $\begin{array}{l}\text { - Hold ICl } \\
\text { - Start } 0.5-1.0 \mathrm{mg} / \mathrm{kg} / \text { day methylprednisolone equivalents PO or } \\
\text { IV once infection has been excluded } \\
\text { Consider permanent discontinuation of ICI if AE worsens or } \\
\text { does not improve. }\end{array}$ & $\checkmark$ \\
\hline 3 & Severe symptoms; limiting self-care ADL & $\begin{array}{l}\text { - Permanently discontinue ICI } \\
\text { - Start } 1-2 \mathrm{mg} / \mathrm{kg} / \text { day methylprednisolone equivalents IV and } \\
\text { prophylactic antibiotics } \\
\text { - Consider plasmapheresis if no improvement or symptoms } \\
\text { worsen after } 3 \text { days }\end{array}$ & $\checkmark$ \\
\hline 4 & $\begin{array}{l}\text { Life-threatening consequences; urgent } \\
\text { intervention indicated }\end{array}$ & $\begin{array}{l}\text { - Permanently discontinue ICI } \\
\text { - Start } 1-2 \mathrm{mg} / \mathrm{kg} / \text { day methylprednisolone equivalents IV and } \\
\text { prophylactic antibiotics } \\
\text { - Consider plasmapheresis if no improvement or symptoms } \\
\text { worsen after } 3 \text { days } \\
\text { - Contact intensive care unit }\end{array}$ & $\begin{array}{l}\checkmark \\
\text { and contact intensive } \\
\text { care unit }\end{array}$ \\
\hline
\end{tabular}

Notes: CTCAE provides grading criteria for encephalopathy, leukoencephalopathy, and reversible posterior leukoencephalopathy syndrome (PRES). For all these irAEs, ICl therapy may be continued for grade 1 irAEs. However, $\geq$ grade 2 events require an ICI hold, and referral to neurology. For events of $\geq$ grade 3 severity, ICl should be permanently discontinued, IV corticosteroids administered, and plasmapheresis considered if there is no improvement, or symptoms worsen, after 3 days.

Peripheral motor and sensory neuropathy

\section{Grade CTCAE Description \\ 1 See CTCAE for grade definitions for each disorder}

Management

- Continue IC

- Consider permanent discontinuation of ICl if AE worsens or does not improve

- Hold ICl

- Refer to neurology

- Consider permanent discontinuation of ICI if AE worsens or does not improve

- Permanently discontinue ICI

- Start 1-2 mg/kg/day methylprednisolone equivalents IV, and prophylactic antibiotics 
Table 3 Recommended management of CTCAE-based immune-related adverse events due to immune checkpoint inhibitor (ICI) therapy (Continued)

Notes: CTCAE provides grading criteria for peripheral motor neuropathy and sensory motor neuropathy. For all these irAEs, ICl therapy may be continued for grade 1 irAEs. However, $\geq$ grade 2 events require an $\mathrm{ICl}$ hold and referral to neurology. For events of $\geq$ grade 3 severity, ICl therapy should be permanently discontinued and IV corticosteroids administered.

\section{OPHTHALMOLOGIC}

Uveitis

Grade CTCAE Description

1 Asymptomatic; clinical or diagnostic observations only

2 Anterior uveitis; medical intervention indicated

3 Posterior or pan-uveitis (Note 1)

Blindness (20/200 or worse) in the affected eye

Management

- Continue ICl

- Ophthalmology referral within 1 week

- Start lubrication drops (artificial tears)

- Hold $\mathrm{ICl}$

- Ophthalmology referral within 2 days, prior to initiating uveitis treatment

- Coordinate treatment with ophthalmologist (topical corticosteroids, cycloplegic agents, systemic corticosteroids)

- Permanently discontinue ICI

- In carefully selected cases it may be appropriate to restart treatment, cautiously, depending on severity, systemic response to immunotherapy and ocular response to topical, local or systemic prednisone (prescribed in coordination with ophthalmologist)

- URGENT ophthalmology referral (preferably uveitis specialist) prior to initiating treatment. Co-ordinate treatment with specialists

- Consider systemic corticosteroids in addition to intravitreal/ periocular corticosteroids/topical corticosteroid treatment as recommended by ophthalmologist

- Permanently discontinue $\mathrm{ICI}$

- URGENT ophthalmology referral (preferably uveitis specialist) prior to initiating any treatment. Co-ordinate treatment with specialists

- Consider systemic corticosteroids in addition to intravitreal /periocular corticosteroids/topical corticosteroid treatment as recommended by ophthalmologist

Note: Unlike anterior uveitis, posterior uveitis can be asymptomatic but nonetheless proceed to visual loss.

Episcleritis

\section{Grade CTCAE Description} observations only

Symptomatic, limiting instrumental ADL moderate decrease in visual acuity (20/40 or better)

3 Symptomatic, limiting self- care ADL; marked decrease in visual acuity (worse than 20/40)
Blindness (20/200 or worse) in the affected eye

\section{Management}

- Continue IC

- Ophthalmology referral within 1 week

- Start lubrication drops (artificial tears)

- Hold ICl

- Ophthalmology referral within 2 days, prior to initiating uveitis treatment

- Coordinate treatment with ophthalmologist (topical steroids, cycloplegic agents, systemic steroids) (See Note)

- Permanently discontinue $\mathrm{ICl}$

- In carefully selected cases it may be appropriate to restart treatment, cautiously, depending on severity, systemic response to immunotherapy and ocular response to topical, local or systemic prednisone (prescribed in coordination with ophthalmologist)

- URGENT ophthalmology referral (preferably uveitis specialist) prior to initiating treatment (See Note). Co-ordinate treatment with specialists.

- Consider systemic steroids in addition to intravitreal /periocular steroids /topical steroid treatment as recommended by ophthalmologist

- Permanently discontinue ICI

- URGENT ophthalmology referral (preferably uveitis specialist) prior to initiating any treatment (See Note). Co-ordinate treatment with specialists. 
Table 3 Recommended management of CTCAE-based immune-related adverse events due to immune checkpoint inhibitor (ICI) therapy (Continued)

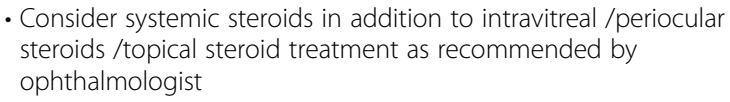

Notes: IMPORTANT: Starting treatment with steroids prior to conducting an eye exam may worsen ocular conditions that are due to infection (e.g., herpetic keratitis/uveitis) or may mask accurate diagnosis and severity grading when the patient is examined by an ophthalmologist.

Blepharitis

Grade CTCAE Diagnosis

Not defined in CTCAE

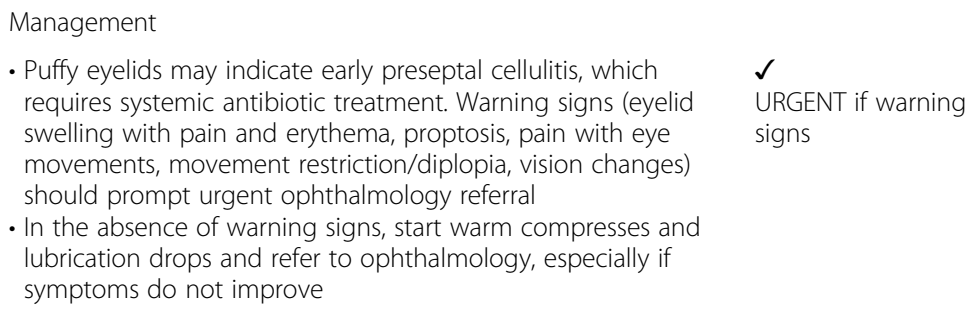

$\checkmark$

URGENT if warning signs

When to refer In cases of non-urgent or emergent referral, photographic documentation is recommended when a new dermatologic manifestation appears, prior to implementing treatment. This facilitates later classification of the $\mathrm{AE}$ when necessary. A same-day dermatology consult is warranted in any patient with blisters covering $\geq 1 \%$ body surface area (BSA), a rash with mucosal involvement, any rash covering $\geq 30 \%$ BSA, and rash with skin pain with or without blisters (excluding dermatomal varicella zoster). For these latter cases, skin biopsy is recommended to help classify the event. Non-acute dermatology referral is recommended for rashes where diagnosis is unclear, grade 2 rash that is worsening, erythema multiforme, blistering disorders of any BSA or for a rash consistent with psoriasis or lichenoid dermatitis that has not responded to topical intervention. Any grade 3 dermatologic toxicity warrants a same-day dermatology consult. Patients with suspected SJS/TEN, severe mucocutaneous reactions characterized by epidermal necrosis and detachment, should be hospitalized immediately and a dermatologist consulted for administration of systemic immunosuppression.

The recommended management of common dermatologic irAEs is presented in Table 3; recommendations for managing uncommon dermatologic irAEs is presented in Additional file 1:Table S1.

\section{Gastrointestinal adverse events Clinical presentation and epidemiology Colitis}

Diarrhea is one of the most frequently reported irAEs in patients taking ICIs. Mild, transient, self-limited diarrhea that occurs on initiation of an immune response should be distinguished from other presentations. Onset occurs after an average of three infusions [11], although it may occcur as soon as following the first infusion. Incidence is higher among patients taking combination anti-CTLA-4/ anti-PD-1 therapy (44\%) than those receiving anti-CTLA$4(23-33 \%)$ or anti-PD-1 ( $\leq 19 \%)$ monotherapy. The combinatorial approach is also associated with increased risk of grade $3 / 4$ symptoms compared with monotherapy, and the proportion of patients experiencing high-grade symptoms is greater with ipilimumab than anti-PD-1 or antiPD-L1 agents [15, 40, 41]. The presence of diarrhea in conjunction with abdominal pain, rectal bleeding, mucus in the stool, and fever should alert the clinician to the possibility of colitis, a potentially serious or even lifethreatening gastrointestinal (GI) complication of ICI therapy. Reports differ on the primary location of ICI colitis, with some finding a uniform distribution [42], and others observing that inflammation preferentially affects the descending colon [43,44], although this may be due to less frequent examination of the proximal colon [44, 45]. Diarrhea and/or colitis may recur months after discontinuation of immunotherapy and can mimic chronic inflammatory bowel disease (IBD) [42, 46].

\section{Hepatitis}

Less frequently observed, but nonetheless well-recognized in patients treated with ICIs, is a typically asymptomatic immune-related hepatitis characterized by elevated alanine aminotransferase (ALT) or aspartate aminotransferase (AST), with or without raised bilirubin. Median onset of transaminase elevation is approximately 6-14 weeks after starting ICI treatment [28]. A minority of patients present with fever. The incidence of any-grade hepatic enzyme disturbance with ipilimumab $3 \mathrm{mg} / \mathrm{kg}$ monotherapy is $<4 \%$ and up to $15 \%$ when dosed at $10 \mathrm{mg} / \mathrm{kg}[24,47]$. Incidence of hepatitis in patients treated with anti-PD-1 ICIs is approximately $5 \%$, but this rises to $30 \%$ in patients treated with combination ipilimumab and nivolumab [13, 28]. 
Acute pancreatitis has been reported but is rare [42]; asymptomatic elevation of lipase and amylase are more common. The role of the gut microbiome in determining treatment response and risk of toxicities, including colitis, in patients treated with ICIs is an area of active investigation.

\section{Diagnostic evaluation}

In the setting of acute diarrhea, initial evaluation should exclude an infectious etiology (consider stool culture, Clostridium difficile, cytomegalovirus (CMV) DNA polymerase chain reaction (PCR), stool ova and parasites). Inflammatory markers (fecal leukocytes/lactoferrin, fecal calprotectin) and fecal occult blood test (FOBT) may help indicate whether there is an inflammatory process underlying the diarrhea. Screening tests for tuberculosis, human immunodeficiency virus (HIV) and hepatitis A and $B$ should be considered if there is potential for use of systemic immunosuppression e.g. infliximab in the near future. Based on the IBD literature, risk of hepatitis $\mathrm{C}$ exacerbation is minimal; as a result, testing for hepatitis $\mathrm{C}$ is not recommended [48, 49].

\section{Colitis}

Radiologically, two distinct patterns of anti-CTLA-4-associated colitis have been observed on computed tomography (CT) imaging: a more common diffuse colitis characterized by mesenteric vessel engorgement, and a segmental colitis with moderate wall thickening and associated pericolonic fat stranding in a segment of preexisting diverticulosis [50]. A fluorodeoxyglucose positron emission tomography (FDG-PET)/CT study can also demonstrate new FDG-avid diffuse colonic wall thickening in patients with immune-related colitis [50]. Colonoscopy is the most accurate means of evaluating the extent and severity of colitis and is recommended in appropriate cases since recent data suggest that the presence of ulceration on endoscopy predicts steroid-refractory disease [51]. For grade $\geq 2$ diarrhea, systemic immunosuppression should be initiated promptly after ruling out infectious etiology. Colonoscopy can be considered if deemed clinically necessary, although it is worth noting that certain types of colitis may have a normal endoscopic appearance, with significant inflammatory features on histology. Therefore, routine mucosal biopsies should be performed for histological examination. In addition, pathology with immunohistochemical staining to rule out CMV infection is critical.

Histologically, colitis that follows treatment with antiCTLA-4 antibodies is characterized by neutrophilic inflammation with increased intraepithelial lymphocytes, crypt epithelial cell apoptosis and few or no features of chronicity. Similarly, anti-PD-1-related colitis typically follows one of two patterns: active colitis with apoptosis (active inflammation, neutrophilic crypt micro-abscesses, increased crypt epithelial cell apoptosis, and presence of crypt atrophy/dropout) or lymphocytic colitis (increased intraepithelial lymphocytes in surface epithelium, surface epithelial injury, and expansion of the lamina propria). Pathological changes may also be visible outside the colon in the duodenum, stomach and/or small bowel [52].

\section{Hepatitis}

Liver function testing prior to initiation of ICIs, and again before each cycle of treatment, can help determine patterns of liver enzyme disturbance. Hepatitis following ICI therapy is typically detected on routine serum liver function tests. Other causes of liver damage such as viral infection, alcohol, other medications or cancer progression should be excluded. Other thromboembolic and outflow obstructive etiology should also be excluded through imaging. On radiologic evaluation, ipilimumabassociated hepatitis has been shown to present with non-specific and variable findings according to clinical severity [53]. Hepatomegaly, edema and enlarged lymph nodes in the periportal region, and attenuated liver parenchyma may be evident on CT and MRI. Liver biopsy, only necessary in complicated cases, may reveal predominantly hepatocyte injury (acute hepatitis pattern) with sinusoidal histiocytic infiltrates, central hepatic vein damage and endothelial inflammation similar to autoimmune hepatitis, or predominant bile duct injury (biliary pattern, with portal inflammation) [53, 54]; rarely, fibrin ring granulomas have also been reported [55].

\section{When to refer}

If infectious work-up is negative, diarrhea due to previous immunotherapy exposure should be considered a possible etiology since colitis can wax and wane after an initial episode. Endoscopy and histology may provide further clarification, and the patient should be referred promptly to a gastroenterologist who is experienced managing patients with gastrointestinal adverse events after immunotherapy. There are reports about progression of colitis to chronic IBD long term [56] and such patients should be followed by a gastroenterologist long term.

\section{Endocrine adverse events}

\section{Clinical presentation and epidemiology}

The two most common endocrine irAEs are acute hypophysitis resulting in hypopituitarism (central hypothyroidism, central adrenal insufficiency, hypogonadotropic hypogonadism), and thyroid disease or abnormalities in thyroid function tests (primary hypothyroidism and thyroiditis). Other endocrinopathies such as primary adrenal insufficiency, T1DM, hypercalcemia, and hypoparathyroidism have been reported but are rare. The prevalence of these disorders varies greatly. This may be due to the non-specific 
presenting signs and symptoms, such as, headache, fatigue, anorexia and nausea, coupled with the fact that hormonal abnormalities are not uncommon in patients with advanced cancer. Diagnosis is also complicated by the fact that baseline screening for endocrine abnormalities is not routinely performed (other than thyroid function tests, in some cases), and corticosteroids may be initiated empirically for suspected irAEs, which interferes with subsequent endocrine testing. A low threshold of clinical suspicion is therefore warranted and, in the absence of alternate etiologies, a diagnostic work-up for endocrine dysfunction should be initiated.

\section{Diagnostic evaluation}

Routine monitoring for clinical signs and symptoms of endocrinopathies, and patient education, are recommended. All patients should be tested before starting treatment for thyroid (thyroid-stimulating hormone [TSH] and free thyroxine [freeT4]), early morning adrenal (adrenocorticotropic hormone $[\mathrm{ACTH}]$ and cortisol) function, and glycemic control (glucose and glycated hemoglobin [HbA1c]). In situations where new elevation in glucose is noted, testing for blood or urinary ketones should be considered. Before each cycle, thyroid testing TSH and free T4) should be repeated, along with a baseline metabolic panel to allow monitoring of glycemic trends.. Routine monitoring with early morning ACTH and cortisol levels should be considered (every month for 6 months, then every 3 months for 6 months then every 6 months for 1 year).

\section{Hypophysitis}

Hypophysitis is most commonly seen with anti CTLA-4 antibody monotherapy (ipilimumab, with an incidence of $\leq 10 \%$ at a dose of $3 \mathrm{mg} / \mathrm{kg}$ and up to $17 \%$ at $10 \mathrm{mg} / \mathrm{kg}$ ), and with combination ipilimumab/nivolumab (incidence $\leq 13 \%$ ) $[10,13,16,17,57]$. The median time from starting ipilimumab to diagnosis of hypophysitis is 8-9 weeks, or after the third dose of ipilimumab $[15,58]$. Symptoms commonly include headache (85\%) and fatigue (66\%); visual changes are uncommon. Clinical suspicion of hypophysitis is frequently raised when routine thyroid function testing shows a low TSH with low free T4, suggestive of a central etiology. Patients have various degrees of anterior pituitary hormonal deficiency, with central hypothyroidism being most commonly seen (>90\%), followed by central adrenal insufficiency, which is also found in the majority of patients [59-61]. Both central hypothyroidism and adrenal insufficiency occur in $>75 \%$ of patients and approximately $50 \%$ of patients present with panhypopituitarism (adrenal insufficiency plus hypothyroidism plus hypogonadism) [61-63]. On magnetic resonance imaging (MRI) of the sella, pituitary enlargement can precede the development of clinical and biochemical evidence of disease. MRI abnormalities, such as stalk thickening, suprasellar convexity, heterogeneous enhancement, and increased height of the gland as compared with baseline scans (when available) are present in most patients at the time of diagnosis. Resolution of pituitary enlargement is common, with all cases resolved on follow up scans after two months $[60,64]$.

All patients with suspected hypophysitis based on clinical findings (headache, fatigue) or biochemical evaluation (routine thyroid function testing showing low free T4 with low/normal TSH) should undergo further testing for diagnostic confirmation. Recommended tests, preferably conducted in the morning around $8 \mathrm{am}$, include thyroid function (TSH, free T4), adrenal function (ACTH, cortisol or $1 \mathrm{mcg}$ cosyntropin stimulation test), gonadal hormones (testosterone in men, estradiol in women), follicle-stimulating hormone [FSH], luteinizing hormone $[\mathrm{LH}]$ ) and MRI of the sella, with pituitary cuts. This should be done prior to administration of steroids. Strict criteria for diagnostic confirmation of hypophysitis are not currently available. Proposed confirmation criteria include $\geq 1$ pituitary hormone deficiency (TSH or ACTH deficiency required) combined with an MRI abnormality, or $\geq 2$ pituitary hormone deficiencies (TSH or $\mathrm{ACTH}$ deficiency required) in the presence of headache and other symptoms.

Management of confirmed hypophysitis includes replacement of deficient hormones (physiologic doses of steroids and thyroid hormone). In the presence of both adrenal insufficiency and hypothyroidism, steroids should always be started prior to thyroid hormone in order to avoid an adrenal crisis. High doses of steroids are necessary in the setting of severe headaches, vision changes or adrenal crisis. Both adrenal insufficiency and hypothyroidism appear to represent long term sequelae of hypophysitis and lifelong hormonal replacement is needed in most cases [59, 64-66]. All patients with adrenal insufficiency should be instructed to obtain and carry a medical alert bracelet.

\section{Thyroid dysfunction}

Thyroid dysfunction (hypothyroidism, hyperthyroidism, and thyroiditis) was reported in $6-20 \%$ of patients in large phase 3 clinical trials.

\section{Hypothyroidism}

Patients with unexplained fatigue, weight gain, hair loss, cold intolerance, constipation, depression and other recognized symptoms should be suspected of having hypothyroidism. Lab tests showing high TSH and low free $\mathrm{T} 4$ are indicative of biochemical hypothyroidism and, if present, additional testing for thyroid antibodies such as thyroid peroxidase (TPO) antibody is warranted. Patients with confirmed hypothyroidism should be started 
on thyroid hormone, with repeat TSH and free T4 levels evaluated 6-8 weeks later. Once a maintenance dose is identified (TSH within normal range) clinical and biochemical re-evaluation should be undertaken every 12 months.

\section{Thyrotoxicosis}

Thyrotoxicosis (high free T4 or total T3 with low or normal TSH) may occur secondary to thyroiditis or Graves' disease. Thyroiditis is the most frequent cause of thyrotoxicosis and is seen more commonly with anti-PD1/PD-L1 drugs than with anti-CTLA-4 agents; Graves' disease is very rare and occurs more commonly with anti-CTLA-4 drugs. Thyrotoxicosis due to thyroiditis may present with weight loss, palpitations, heat intolerance, tremors, anxiety, diarrhea and other symptoms of hypermetabolic activity, although these symptoms may be masked if the patient is taking beta-blockers. Most commonly, patients are asymptomatic (painless thyroiditis) and routine laboratory monitoring shows high free T4 or triiodothyronine (T3) levels, with low/normal TSH. A thyrotoxic phase occurs an average of one month after starting the drug. Additional tests can be undertaken when thyroiditis is suspected, primarily to rule out other causes of thyrotoxicosis such as Graves' disease. These include thyroid stimulating hormone receptor antibody [TRAb] or thyroid stimulating immunoglobulin (TSI) and TPO as well as images when feasible: radioactive iodine uptake scan (RAIUS) or Technetium (Tc)-99 m [pertechnetate] thyroid scan if recent iodinated contrast was used. Thyroiditis is a self-limiting process and leads to permanent hypothyroidism after an average of 1 month after the thyrotoxic phase and 2 months from initiation of immunotherapy. Conservative management during the thyrotoxic phase of thyroiditis is sufficient. Non-selective beta blockers, preferably with alpha receptorblocking capacity, may be needed in symptomatic patients. Repeat thyroid hormone levels should be performed every 2-3 weeks and thyroid hormone replacement initiated at the time of hypothyroidism diagnosis $[59,64]$.

\section{Type 1 diabetes mellitus}

Development of polyuria, polydipsia, weight loss, nausea and/or vomiting should prompt investigation for possible development or worsening of T1DM. Diagnosis and management of T1DM is based on recognized guidelines [67]. Tests for antibodies (glutamic acid decarboxylase [GAD65], anti-insulin, anti-islet cell A, zinc transporter 8 [Zn-T8]), C-peptide and insulin could distinguish between type 1 and type 2 disease.

\section{When to refer}

An endocrinology consultation is recommended in all cases of suspected or confirmed hypophysitis, primary hypothyroidism, hyperthyroidism, thyroiditis, type $1 \mathrm{DM}$ and all rare endocrinopathies.

\section{Pulmonary adverse events Clinical presentation and epidemiology Pneumonitis}

The most common lung toxicity observed in patients receiving ICI treatment is pneumonitis. The overall incidence of pneumonitis associated with PD-1/PDL-1 and CTLA-4-targeted therapies is $<5 \%$, with high-grade ( $\geq$ grade 3) events occurring in 1-2\% of patients. Higher rates have been reported for combinations of PD-1 and CTLA-4 inhibitors [68]. These numbers are not clinically trivial, as pneumonitis is one of the most common causes of ICI-related death. Moreover, the incidence of pneumonitis is increasing as therapeutic indications for ICIs expand, and more complex regimens are developed. Pneumonitis may present on imaging studies as cryptogenic organizing pneumonia (COP), nonspecific interstitial pneumonitis (NSIP), hypersensitivity pneumonitis (HP), or usual interstitial pneumonitis (UIP)/pulmonary fibrosis (PF). Clinical and radiographic findings of ICI-related pneumonitis may closely mimic pneumonia, lymphangitic spread of disease, cancer progression, and diffuse alveolar hemorrhage. The radiographic appearance of pneumonitis may be clinically asymptomatic or, alternatively, associated with new or worsening shortness of breath, cough, wheezing, chest pain, reduced exercise tolerance, fatigue with activities of daily living (ADL) and new or increasing requirement for supplementary oxygen. Acuity of onset and severity may also vary, suggesting the importance of vigilance and rapid response in some cases. Studies have suggested a higher incidence of any grade $(3.6 \%$ vs. $1.3 \%)$ and severe (1.1\% vs. $0.4 \%)$ pneumonitis with PD-1 inhibitors compared with PD-L1 inhibitors [69]. Combination therapies with anti-CTLA-4/anti-PD-1/PD-L1 immunotherapy and with $\mathrm{ICI} /$ cytotoxic combinations also confer a higher risk of pneumonitis versus ICI monotherapy $[68,70]$. Higher rates of pneumonitis have also been reported among ICI-treated patients with non-small cell lung cancer (NSCLC) compared to patients with melanoma [71]. Pneumonitis onset appears earlier in cases of NSCLC (median [range]: 2.1 [0.2-27.4] months) versus melanoma (median [range]: 5.2 [0.2-18.1] months) [72]. IrAEs associated with other organ systems, including hepatitis, colitis, duodenitis, esophagitis, thyroiditis, hypophysitis, arthritis, myositis, vitiligo, nephritis, and anemia may occur in up to $50 \%$ of patients and confound therapy. These irAEs may occur concomitantly, precede or follow the development of pneumonitis. In patients with preexisting lung diseases, such as chronic obstructive pulmonary disease (COPD) or $\mathrm{PF}$, the diagnosis of pneumonitis is particularly challenging and failure to recognize and treat pneumonitis in a timely manner could lead to poor clinical outcomes.

In addition to pneumonitis, ICI therapy has been associated with pleural effusions, pulmonary sarcoidosis and sarcoid-like granulomatous reactions. Sarcoid-like reactions 
have been reported following both CTLA- 4 and PD-1/PDL1-targeted therapies. Increased numbers of $\mathrm{T}$ helper 17 (Th17.1) cells are seen in the bronchoalveolar lavage (BAL) fluid of these patients, suggesting that TH17 cells may play an important role in the pathogenesis of this disease [73]. Sarcoidosis may be asymptomatic or present with cough, wheezing, fatigue and/or chest pain. Data in this area are scant at present, although case reports suggest that the development of sarcoidosis may be associated with prolonged cancer response [74, 75].

Treatment strategies for ICI related pneumonitis, based on pneumonitis grade, are detailed in Table 3. Patients with grades 1-2 pneumonitis may be managed as outpatients while those with pneumonitis grade 3 or higher typically require hospitalization. Drug withdrawal is the mainstay of treatment for pneumonitis of all grades. For patients with grade 1 pneumonitis, re-challenge following resolution of infiltrates and close follow-up is reasonable. In these patients, symptoms should be monitored every $2-3$ days. A repeat chest CT should be performed prior to the next scheduled dose of ICI and if the infiltrates have resolved, ICI therapy may be cautiously resumed with close followup. Bronchoscopy should be considered for evidence of new or persistent infiltrates. Patients with grade 2 or higher pneumonitis may require oral/intravenous corticosteroids. Recrudescence of pneumonitis signs and symptoms has been reported following rapid steroid taper; a minimum 4-6 week taper is therefore recommended. Additional immunosuppression with infliximab and/or cyclophosphamide is warranted among patients with recalcitrant disease.

\section{Sarcoidosis}

Once a diagnosis of sarcoidosis is established, immunotherapy should be withheld, particularly in patients with extensive disease (stage $\geq 2$ ), extrapulmonary disease involving critical organ systems (ocular, myocardial, neurologic, renal), or sarcoid-related hypercalcemia. Treatment for irAE-related sarcoidosis should be considered if there is 1) progressive radiographic change; 2) persistent and/or troublesome pulmonary symptoms; 3 ) lung function deterioration (total lung capacity (TLC) decline of $\geq 10 \%$, forced vital capacity (FVC) decline of $\geq 15 \%$; diffusing capacity of the lungs for carbon monoxide (DLCO) decline of $\geq 20 \%$ ); 4) concomitant involvement of critical extrapulmonary organ systems; or 5) sarcoid-related hypercalcemia. These guidelines are extrapolated from standard management guidelines for sarcoidosis in the general population. Further investigations of sarcoidosis management in the ICI setting are needed.

\section{Diagnostic evaluation Pneumonitis}

The diagnosis of pneumonitis is suggested by the presence of new or progressive pulmonary infiltrates and ground glass changes on lung imaging studies. The infiltrates are typically bilateral, but may be asymmetric. CT imaging is more reliable than chest radiographs in identifying these changes, and is the imaging modality of choice. Baseline and ongoing oxygen saturation (at rest and on ambulation) should be monitored in all patients, as well as chest CT, pulmonary function tests (PFTs), and a 6-min walk test (6MWT). A pulmonology consult is warranted in any patient with suspected pneumonitis. Atypical symptoms such as fever and productive cough should also trigger an infectious disease consultation. Fiberoptic bronchoscopy with BAL may be helpful in excluding competing diagnoses. Lung biopsies are typically not warranted, but may be useful in the setting of suspicious lesions and unexplained lymphadenopathy.

\section{Sarcoidosis}

The diagnosis of pulmonary sarcoidosis is suggested by radiographic evidence of intrathoracic lymphadenopathy and irregular densities, coupled with histologic evidence of epithelioid non-caseating granulomas obtained from endobronchial ultrasound (EBUS), fine needle aspiration (FNA) or transbronchial lung biopsy (TBBx). Since sarcoidosis can mimic malignant disease progression, both clinicians and radiologists should be aware of this possibility. Confirmation requires exclusion of infections and other competing diagnoses. Patients may also present with extrapulmonary manifestations of sarcoidosis. Therefore, once the diagnosis is established an eye examination and baseline electrocardiogram should be considered to investigate involvement of other organ systems. The natural history of irAE-related sarcoidosis is not known and treatment strategies for sarcoid in this setting have not been established.

\section{When to refer}

Referral to a pulmonary specialist for bronchoscopy should be pursued in all patients with radiographic and/or clinical evidence of pneumonitis. Such evidence includes new pulmonary infiltrates on lung imaging, or new or worsened hypoxemia, dyspnea or cough. Unexplained lymphadenopathy or atypical pulmonary nodules and densities should also prompt a pulmonary referral. Infectious disease consultation should be considered for patients with $\geq$ grade 2 pneumonitis. Long-term specialist follow-up is also advisable in any patient with confirmed immunerelated lung disease.

\section{Rheumatologic/musculoskeletal adverse events Clinical presentation and epidemiology}

Recognizing rheumatologic and musculoskeletal irAEs in the oncology setting is challenging due to the broad range of potential presenting symptoms and the prevalence of musculoskeletal complaints in the general population. 
Although a paucity of epidemiological data limits our understanding of the true incidence of these irAEs, they are increasingly reported across care settings. Since delayed diagnosis and treatment can lead to long-term disability, and disorders may become chronic and require ongoing immunosuppressive/immunomodulatory therapy, it is important to understand typical symptom presentation and recommended management. Preserving quality of life and ability to perform ADL is a priority.

One of the most commonly reported rheumatologic irAEs is an inflammatory oligo or polyarthritis that can lead to rapid joint damage and may persist after discontinuation of immunotherapy. Arthralgia has been reported in approximately $15 \%$ of patients receiving ICIs, but the incidence of inflammatory arthritis, which is typically grade 2 or less, has not yet been systematically reported [76]. Arthritis is rarely the sole irAE, with most patients having other organ systems involvement. In a small series, the median time to onset was five months after starting ICI therapy. Clinically, three phenotypes have been described: 1) predominantly large joint reactive arthritis that, on occasion, develops in association with conjunctivitis and uveitis; 2) polyarthritis resembling rheumatoid-like arthritis, affecting the small joints of the hand (metacarpophalangeal [MCP], proximal interphalangeal [PIP] joints or wrist), rarely associated with typical rheumatoid factor (RF) or anti-citrullinated protein antibodies (ACPA), but potentially erosive; and 3) seronegative, oligo and polyarthritis, typically starting in the medium/large joints, characterized by synovitis and involvement of tendons and entheses, with or without joint erosions. Combination anti-CTLA-4/anti-PD-1 therapy is associated with a greater risk of arthritis than monotherapy, although incidence is unaffected by drug or type of malignancy. Management often requires moderate-dose corticosteroids, sometimes in conjunction with steroidsparing immunomodulators and disease-modifying antirheumatic drugs (DMARDs) including tumor necrosis factor inhibitors (TNFi), methotrexate, leflunomide, sulfasalazine, and hydroxychloroquine. Persistence of inflammatory arthritis up to two years after discontinuation of ICIs has been seen, with ongoing requirement for immunomodulatory therapy. Beyond arthritis, less commonly reported rheumatologic irAEs recognized in the context of ICI therapy include sicca, with severe eye and mouth dryness, and parotitis; inflammatory myositis, most commonly resembling polymyositis, occasionally resulting in rhabdomyolysis; vasculitides including giant cell arteritis (GCA) and polymyalgia rheumatica (PMR); systemic lupus erythematosus (SLE) and sarcoidosis [76, 77].

One of the primary difficulties in ensuring accurate reporting of rheumatologic irAEs is the nature of severity grading in the current CTCAE. The current version (version 4) classifies many clinically significant rheumatologic events that require corticosteroids or immunomodulatory treatment as grade $1 / 2$, whereas the rheumatology CTCAE (rCTCAE) compiled by the Outcome Measures in Rheumatology network (formerly Outcome Measures in Rheumatoid Arthritis Clinical Trials; OMERACT) (RCTCAE version 2.0) [34] classifies similar symptoms one or two severity grades higher. Of particular importance, the current CTCAE classifies impairment in instrumental ADLs (taking medications, preparing meals, housework, using transportation) as grade 2, despite the fact that this represents a considerable degree of functional disability and loss of independence. This also has implications for the detection of "clinically significant" musculoskeletal irAEs in clinical trial databases.

Current CTCAE terms for musculoskeletal symptoms (e.g. arthritis and myositis) are not easily converted to clinically relevant descriptors. Lack of precision may result in diffusion of an irAE signal, distorting the epidemiological landscape. For example, oncologists must choose between several different codes to document a swollen joint (joint effusion, joint pain, joint function, arthritis) or muscle weakness (myalgia, muscle weakness, change in lower extremity function). As such, it may be more appropriate to aggregate similar coding subtypes to better reflect the true incidence of musculoskeletal irAEs.

\section{Diagnostic evaluation}

A diagnostic algorithm for inflammatory arthritis has recently been reported [78]. The SITC Toxicity Management Working Group evaluated and discussed this algorithm and made suggestions for its modification. The revisions are noted below.

Grade 1: Joint examination (swelling/tenderness), functional assessment, consider rheumatology referral, especially if symptoms persist.

Grade 2/3: Joint examination, functional assessment, consider laboratory testing (antinuclear antibody [ANA] rheumatoid factor $[R F]$, cyclic citrullinated peptide antibody [anti-CCP], erythrocyte sedimentation rate [ESR]/ $\mathrm{C}$-reactive protein $[\mathrm{CRP}]$ ). Consider imaging (plain $\mathrm{X}$ ray of affected joints, joint MRI and/or musculoskeletal ultrasound).

\section{When to refer}

All patients with CTCAE $\geq$ grade 2 inflammatory arthritis should be referred to rheumatology. Also consider referring any patient whose symptoms persist for $>6$ weeks or who requires $>20 \mathrm{mg}$ prednisone (or equivalent) daily that cannot be tapered to $<10 \mathrm{mg} /$ day within 4 weeks [78]. All patients with suspected myositis, presenting with muscle weakness and elevated creatine kinase (CK), should be referred to rheumatology or neurology, as this can be a life-threatening adverse event. 
Because erosive, irreversible joint damage has been seen within weeks of symptom onset, early involvement of rheumatologists is recommended to determine if additional disease-modifying therapy beyond steroids is required.

For other suspected rheumatologic manifestations (e.g. vasculitis, myositis, scleroderma, etc.), rheumatology referral is advisable even if the symptoms are mild, to ensure that appropriate diagnostic testing and optimal management can be coordinated to prevent permanent organ damage.

\section{Infusion reactions}

\section{Clinical presentation and epidemiology}

Infusion reactions may present with constitutional symptoms such as fever, rigor, pruritus, hypotension, dyspnea, chest discomfort, rash, urticaria, angioedema, wheezing or tachycardia, as well as the possibility of anaphylaxis requiring urgent intervention. Infusion reactions (all grades) are reported in $25 \%$ of patients receiving avelumab (premedication with acetaminophen and an antihistamine is recommended) [7] and in less than $10 \%$ of patients receiving other approved immune checkpoints inhibitors [5-9, 79-81]. Infusions of ipilimumab appear to be welltolerated, with a low incidence $(<6 \%)$ of infusion reactions even when the infusion is delivered over $30 \mathrm{~min}$ (as opposed to the standard 90 min timeframe) when patients are pre-medicated with diphenhydramine and/or corticosteroids [82]. Severe/life-threatening infusion reactions occurred in less than $2 \%$ of the patients. Mild to moderate reactions are managed with symptomatic treatment and by reducing the rate or temporarily interrupting the infusion [4-9]. Severe/life-threatening reactions should be managed promptly and in accordance with the institutional guideline for infusion reactions; permanent discontinuation is recommended for such cases (grades 3 or 4) [80].

\section{Diagnostic evaluation}

Infusion reactions are common to many cancer treatments and appropriate training and procedures should be in place while patients are receiving an immunotherapy infusion. The severity of an infusion reaction should be rapidly assessed and appropriate treatment implemented in accordance with the institutional guideline. Life-threatening reactions with hypoxia and/or shock should be aggressively managed [80].

\section{When to refer}

Cancer patients often receive more than one drug during infusion; patients with severe or life-threatening reactions (CTCAE grade 3 or 4) should therefore be referred to an allergist. Appropriate assessment and counseling could prevent future re-exposure to drugs that have previously caused severe reactions.

\section{Uncommon immune-related adverse events Cardiovascular adverse events Clinical presentation and epidemiology}

Cardiac irAEs due to ICIs may present with non-specific symptoms such as fatigue and weakness. However, more typical cardiac symptoms of chest pain, shortness of breath, pulmonary or lower extremity edema, palpitations, irregular heartbeat, rapid onset of heart failure symptoms or new heart block on electrocardiogram (ECG) can occur at any time, more frequently within the first few months of treatment. Other signs and symptoms may include muscle pain or syncope. Patients who develop immune toxicities of other organ systems may also develop cardiovascular toxicities, potentially with symptoms that overlap with myositis (myalgias, rhabdomyolysis) or myocarditis or pericarditis (fever, chest pain with inspiration, diffuse ST elevation on ECG), making accurate diagnosis a considerable challenge. It is suggested that there may be a link between rhabdomyolysis/ myositis, vasculitis and cardiac toxicity. However, myocarditis, pericarditis and cardiac dysfunction due to ICIs are rare and the true incidence is unknown; current estimates suggest less than $1 \%$ of patients [22]. Moreover, due to varying definitions of cardiotoxicity [83], the obscurity of CTCAE entries for some cardiac irAEs, especially myocarditis, and the absence of systematic monitoring or coding mechanism for cardiac events in immunotherapy trials, cardiac irAEs are likely under-reported. In particular, myocarditis is a difficult diagnosis to make in any clinical situation, but especially in a patient being actively treated for cancer [84]. The expert consensus is to have high vigilance for development of cardiac symptoms in all patients, but especially in those with evidence of myocarditis, vasculitis or myositis.

Cardiac irAEs are seen across the ICI drug class, with higher incidence in patients taking combination antiCTLA-4/anti-PD-1 treatment compared to monotherapy. Patients, including those with known cardiac comorbidities, should not be denied therapy with ICIs solely on the basis of the potential for cardiotoxicity, but the level of vigilance has to be raised. The non-specific presentation of cardiac irAEs and potential to cause rapid clinical deterioration with a higher than acceptable rate of mortality with cardiac toxicity, make it imperative to maintain a low threshold for clinical suspicion and early specialist referral.

\section{Diagnostic evaluation}

At baseline, prior to initiating ICI therapy, it is suggested that a judicious combination of biomarkers (e.g., troponin I or T, brain natriuretic peptide [BNP] or N-terminal pro B-type natriuretic peptide [NT pro-BNP], total $\mathrm{CK}$, fasting lipid profile, total $\mathrm{CK}$ and an electrocardiogram [ECG] be evaluated in all patients). Myocarditis is very rare but other potentially serious cardiac manifestations 
(life-threatening rhythm disturbances and acute coronary syndromes) are reported more commonly [85]. Since the major indicator of suspicion for both myocarditis and acute coronary syndrome is elevated troponin, a fasting lipid profile serves as an important screening tool to distinguish between atherosclerosis-related troponin elevation and potential myocarditis. Two-dimensional echocardiography (2-D Echo) may also be warranted in high-risk patients with cardiac history, symptoms of dyspnea, or if initial tests are abnormal. Serial ECGs and cardiac biomarker testing should be considered, particularly in patients with abnormal baseline investigations or suspicious symptoms. There are no current recommendations for the appropriate time interval between tests. Patients who develop concerning symptoms while undergoing ICI therapy should have chest imaging to exclude pulmonary embolism, pneumonitis, or pulmonary edema, as well as an ECG; cardiac biomarkers done at baseline evaluation should be retested. A repeat $2 \mathrm{D}$ Echo should be considered in any patient who has significant dyspnea or abnormal cardiac safety screening tests.

\section{When to refer}

An accurate baseline CV risk assessment should be undertaken, including consultation with a cardiologist if appropriate, in any patient who has multiple CV risk factors or established CV disease at the onset of immune therapy. Immediate referral is warranted for any patient who develops abnormal cardiac test results during the course of ICI therapy. Since myocarditis can rapidly lead to death, patients with suspected or documented myocarditis should be admitted to the hospital for cardiac monitoring. Patients with confirmed myocarditis should receive emergent intervention with high dose corticosteroids, and immediate discontinuation of immunotherapy. Until data are available (e.g., cut-off levels of troponin) to determine when to start corticosteroids in patients with possible (as opposed to confirmed) myocarditis, this decision should be made on a case by case basis. The importance of active, ongoing consultation with a cardiologist to discuss the risk/benefit of continuing ICI therapy, starting steroids, or instituting other cardiac treatments, cannot be overstated.

\section{Hematologic adverse events Clinical presentation and epidemiology}

Although rare, hematologic irAEs have been described following ICI treatment and the literature includes case reports of hemolytic anemia, red cell aplasia, neutropenia, thrombocytopenia, myelodysplasia and hemophilia A $[15,28,86]$. An active hematologic irAE also needs to be distinguished from transient changes in laboratory values that can occur during initiation of an immune response. Post treatment lymphcytosis, eosinophilia, neutrophilia and monocytosis can be observed and are not typically clinically significant though some reports suggest they may be prognostic [87]. Persistent post treatment cytopenias or progressive cytopenias should be evaluated for autoimmune causes as well as with a peripheral smear, reticulocyte count and assessment for hemolysis [88]. Causal attribution is complicated by the fact that malignant disease and its complications can also lead to cytopenias. Since the CTCAE definition of thrombocytopenia describes absolute platelet levels rather than an indication of changes in cell number, it is not a reliable tool for evaluating potentially life-threatening ICI-induced thrombocytopenia.

\section{Diagnostic evaluation}

Complete blood count (CBC) should be monitored at the start of immune therapy, at intervals during treatment, and periodically in long-term survivors who are no longer receiving treatment. Development of anemia should prompt evaluation for common causes such as GI bleeding, cancer-related anemia or cancer progression, or causative drugs, including a work up for hemolysis. If the source of anemia cannot be identified, bone marrow biopsy may be indicated to rule out red cell aplasia. Similarly, any patient who develops thrombocytopenia or neutropenia should be evaluated for potential causes including medication-related cell destruction or disease progression; in cases where an obvious cause cannot be identified, an autoimmune cause should be considered and investigated accordingly.

\section{When to refer}

In general, patients with unexplained cytopenias should be referred to hematology for evaluation.

\section{Renal adverse events}

\section{Clinical presentation and epidemiology}

Overall, renal irAEs are considered rare, occurring in $2 \%$ (ICI monotherapy) to 5\% (combination ipilimumab/nivolumab) of patients taking ICIs, with underlying pathology only beginning to be characterized and reported [89, 90]. Most reports document isolated cases of interstitial nephritis with specific agents and regimens, such as anti-PD-1 monotherapy, and combination anti-CTLA-4/PD-1 treatment, in melanoma $[91,92]$. Nephritis has not been associated with anti-PD-L1 monotherapy to date. Three cases of acute renal failure were also reported during a study of nivolumab and doublet platinum chemotherapy in NSCLC [93]. There are also case reports of lupus nephritis [94] and granulomatous nephritis [95, 96] following ipilimumab treatment, and a single case of nephritis described after treatment with avelumab [97]. However, recent data suggest the incidence of renal irAEs may be under-reported with low-grade kidney injury affecting 25-29\% of patients taking certain ICIs [90]. The onset of renal injury seen with PD-1 
inhibitors usually occurs 3-10 months after initiation of treatment, whereas irAEs secondary to anti-CTLA-4 agents tend to have an earlier onset, after 2-3 months [90]. Renal toxicity from ICIs is usually asymptomatic, although oliguria, hematuria, peripheral edema and anorexia are occasionally reported. Management requires considerable clinical judgment.

\section{Diagnostic evaluation}

Diagnosis of renal impairment may be complicated by concomitant medications that precede, or are prescribed during the course of, immunotherapy treatment. Nonetheless, evidence of gradually rising serum creatinine should prompt clinical suspicion. As such, serum creatinine should be monitored on starting immunotherapy treatment, and at intervals throughout the treatment course. If creatinine remains elevated for $>2-3$ days, monitor weekly (grade 1 ) or every 2-3 days (grade 2 ). It is important to exclude other causes of renal dysfunction through active inquiry about new medications, correction of dehydration and, possibly, additional investigations such as bladder and/or renal ultrasound, urinalysis, assessment of serum electrolytes, or other studies based on history. In suspected cases of immune-related renal disease, renal biopsy should be considered to confirm etiology and guide management. Because renal toxicity typically resolves, treatment can resume if grade 2-3 adverse events resolve promptly, but therapy should be discontinued in the face of persistent or recurrent grade 2-3 adverse events, or emergence of grade 4 toxicity. A nephrology consult should be considered for any persistent $\geq$ grade 3 renal impairment, or for recurrent renal toxicity following a corticosteroid trial.

\section{When to refer}

A nephrology consult should be considered in patients with persistent grade $2-3$ elevation in creatinine, $\geq 3$-fold increase in creatinine over baseline, or whenever there is evidence of metabolic change consistent with renal failure.

\section{Neurologic adverse events}

\section{Clinical presentation and epidemiology}

Neurologic irAEs are uncommon, with an overall incidence of $<4 \%$ following treatment with anti-CTLA-4 antibodies, $6 \%$ with anti-PD-1 antibodies, and $12 \%$ with combination therapy involving both [98]. Most events are mild and present with non-specific symptoms such as headache; irAEs grade 3 or higher occur in $<1 \%$ of patients [98]. Examples of neurologic irAEs include autoimmune encephalitis, myasthenic syndrome/myasthenia gravis, Guillain-Barré syndrome, peripheral sensorimotor neuropathies, Posterior Reversible Encephalopathy Syndrome (PRES), aseptic meningitis and transverse myelitis [99]. Relevant CTCAE terms include encephalopathy, leukoencephalopathy, peripheral motor neuropathy, peripheral sensory neuropathy reversible posterior leukoencephalopathy syndrome, and 'nervous system not otherwise specified'. Common presenting features of autoimmune encephalitis, meningitis and encephalopathy include altered mental status, headache, seizures, focal neurologic abnormalities and PRES.

\section{Diagnostic evaluation}

Diagnostic work-up should include history and physical examination with full neurologic exam in all patients. Evaluation of possible autoimmune encephalitis, meningitis and encephalopathy should include lumbar puncture and brain MRI, with and without contrast; it is important to rule out infection, screen for unsuspected central nervous system (CNS) metastasis and/or leptomeningeal spread. Paraneoplastic syndromes should also be considered. Diagnostic evaluation of suspected peripheral sensorimotor neuropathies should include differential diagnosis of disorders including, but not limited to, diabetic neuropathy and vitamin B12 deficiency. Consider imaging as appropriate, as well as nerve biopsy; this is a diagnosis of exclusion, but in most cases it is a clinical diagnosis.

\section{When to refer}

Neurology consultation is recommended for all neurologic irAEs grade 2 and higher.

\section{Ophthalmologic adverse events}

\section{Clinical presentation and epidemiology}

Ocular irAEs, predominantly uveitis (anterior more commonly than posterior or panuveitis) are reported in $<1 \%$ of patients taking ICIs $[13,15]$. There have also been reports of orbital inflammation, episcleritis, blepharitis, optic nerve swelling, peripheral ulcerative keratitis and Vogt-KoyanagiHarada picture with localized serous retinal detachment [100-102]. Patients prescribed ICIs should be advised to alert the clinician to new onset of blurred vision, floaters, flashing lights, changes in color vision, eye redness, photophobia or light sensitivity, visual distortion and visual field changes, scotomas, tender eyes or pain on eye movement, eyelid swelling or proptosis or double vision. Patient counseling is crucial to ensure that early signs and symptoms are recognized in a timely manner.

\section{Diagnostic evaluation}

Although prompt ophthalmologic referral is important in ALL cases of visual complaints, certain tests can be performed by the oncologist in the office. These include examination for visual acuity, which can be done using an eye chart on a smart phone with the patient wearing reading glasses for near vision or glasses for distant vision, as necessary; color vision; red reflex; pupils (equal, 
round, reactive), including testing for an afferent pupillary defect, which can indicate optic nerve or extensive retinal disease; and penlight inspection of the anterior part of the eye. Direct ophthalmoscopy to examine the optic nerve and retina is unlikely to be useful for diagnosis of retinal or optic nerve issues when performed by a nonophthalmology-trained physician. Ocular irAEs may be asymmetric so it is important to examine each eye separately. Ocular irAEs are frequently accompanied by irAEs in other systems, especially colitis, so broader systems inquiry is helpful.

\section{When to refer}

Complaints of red, painful, dry or irritated eyes, or visual disturbance in a patient taking an ICI should alert the clinician to the need for immediate ophthalmological referral for diagnosis, classification and management, which can be difficult for the oncologist since different ocular pathologies and grades may present with similar symptoms and detailed ophthalmological evaluation needs to be performed by an ophthalmologist, including a slit lamp exam and dilated fundus exam. Sometimes grade 2 or 3 severity irAEs may only present with asymptomatic or mild changes in vision, and time to ophthalmology access can vary depending on the setting (academic versus community hospital). Clinical suspicion and prompt referral are therefore essential. Starting systemic or topical treatment with corticosteroids prior to conducting an eye exam should be avoided unless systemic steroids are indicated for a concurrent, non-ophthalmological toxicity, since it may worsen ocular conditions that are due to infection (e.g., herpetic keratitis/uveitis) or may mask accurate diagnosis and severity grading when the patient is examined by an ophthalmologist. Urgent referral is warranted for any grade 3 or 4 irAEs, but even patients with grade 1 or 2 toxicities should undergo full ophthalmological evaluation, proper grading, work up and treatment evaluation by an ophthalmologist within a few days. Puffy eyelids may indicate early preseptal cellulitis, which requires systemic antibiotic treatment. Warning signs (eyelid swelling with pain and erythema, proptosis, pain with eye movements, movement restriction/diplopia, vision changes) should prompt urgent ophthalmology referral.

\section{Conclusions}

As the number of patients treated with checkpoint inhibitors grows, and the volume of real-world data increases, the etiology and characterization of immunotherapyrelated toxicities will become clearer, and management more targeted and effective. Since adverse events may occur late, even after terminating active treatment, and there is a potential for long-term chronic complications, constant vigilance and early recognition and treatment of immune-related adverse events is important. Until prospective clinical data are available, the consensus recommendations provided here, on the diagnosis and management of immune checkpoint inhibitor-related adverse events, will hopefully serve as a starting point to help clinicians provide timely and effective management of immune-related toxicities in their patients with cancer.

\section{Additional file}

Additional file 1: Table S1. Recommended management of uncommon dermatologic immune-related adverse events. (DOCX $24 \mathrm{~kb}$ )

\section{Abbreviations}

2-D echo: Two-dimensional echocardiogram/echocardiography; 6MWT: 6 min walk test; AACR: American Association for Cancer Research; ACCC: Association of Community Cancer Centers; ACPA: Anti-citrullinated protein antibodies; ACTH: Adrenocorticotropic hormone; ADCC: Antibodydependent cell-mediated cytotoxicity; ADL: Activities of daily living; ALT: Alanine aminotransferase; ANA: Antinuclear antibody; Anti-CCP: Cyclic citrullinated peptide antibody; Anti-RF: Anti-rheumatoid factor (anti-RF); Anti-TNF: Anti-tumor necrosis factor; ASCO: American Society of Clinical Oncology; AST: Aspartate aminotransferase; ATG: Anti-thymocyte globulin; BAL: Bronchoalveolar lavage; BID: Two times daily; BNP: B-type natriuretic peptide; BSA: Body surface area; CBC: Complete blood count; CK: Creatine kinase; CMP: Complete metabolic panel; CMV: Cytomegalovirus; CNS: Central Nervous System; COP: Cryptogenic organizing pneumonia; COPD: Chronic obstructive pulmonary disease; CRP: C-reactive protein; CT: Computed tomography; CTCAE: Common Terminology Criteria for Adverse Events; CTLA-4: Cytotoxic T lymphocyte-antigen-4; DIC: Disseminated intravascular coagulation; DLCO: Diffusing capacity of the lungs for carbon monoxide; DMARDS: Disease modifying anti-rheumatic drugs; DRESS: Drug rash with eosinophilia and systemic symptoms; EBUS: Endobronchial ultrasound; ECG: Electrocardiogram; ESR: Erythrocyte sedimentation rate; FDA: U.S. Food and Drug Administration; FDG-PET: Fluorodeoxyglucose positron emission tomography; FNA: Fine needle aspiration; FOBT: Fecal occult blood test; FreeT4: Free thyroxine; FSH: Follicle-stimulating hormone; FVC: Forced vital capacity; GAD65: Glutamic acid decarboxylase; GCA: Giant cell arteritis; Gl: Gastrointestinal; HbA1c: Glycated hemoglobin; HBcAb: Hepatitis B core antibody; HBsAb: Hepatitis B surface antibody; HBsAg: Hepatitis B surface antigen; HCAb: Hepatitis C antibody; Hgb: Hemoglobin; HIV: Human immunodeficiency virus; HP: Hypersensitivity pneumonitis; HRT: Hormone Replacement Therapy; HSV: Herpes simplex virus; IBD: Inflammatory bowel disease; ICls: Immune checkpoint inhibitors; ICU: Intensive care unit;

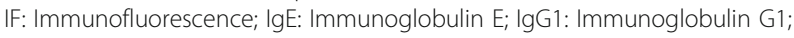
IgG4: Immunoglobulin G4; irAEs: Immune-related adverse events;

IVIG: Intravenous immunoglobulin; LH: Luteinizing hormone; LLN: Lower limit of Normal; mAbs: Monoclonal antibodies; MCP: Metacarpophalangeal; MedDRA: Medical Dictionary for Regulatory Activities; MRI: Magnetic resonance imaging; NCCN: National Comprehensive Cancer Network; $\mathrm{NCl}$ : National Cancer Institute; NIH: National Institutes of Health; NSCLC: Non-small cell lung carcinoma; NSIP: Nonspecific interstitial pneumonitis; NT pro-BNP: N-terminal pro B-type natriuretic peptide; ONS: Oncology Nursing Society; PASI: psoriasis area severity index; PCR: Polymerase chain reaction; PD-1: Programmed cell death protein-1; PD-L1: Programmed cell deathligand 1; PF: Pulmonary fibrosis; PFTs: Pulmonary function tests; PIP: Proximal interphalangeal; PMR: Polymyalgia rheumatic; PRES: Posterior Reversible Encephalopathy Syndrome; QID: Four times daily; RA: Rheumatoid arthritis; RAIUS: Radioactive iodine uptake scan; SITC: Society for Immunotherapy of Cancer; SJS/TEN: Stevens-Johnson Syndrome/toxic epidermal necrolysis; SLE: Systemic lupus erythematosus; T1DM: Type I Diabetes; T3: Triiodothyronine; TBBx: Transbronchial lung biopsy; Tc: Technetium; Th17.1: T helper 17 cells; TLC: Total lung capacity; TNFi: Tumor necrosis factor inhibitor; TPO: Thyroid peroxidase; TRAb: Thyroid-stimulating hormone receptor antibody; TSH: Thyroid-stimulating hormone; TSI: Thyroid-stimulating immunoglobulin; UIP: Usual interstitial pneumonitis; ULN: Upper Limit of Normal; UVB: Short wave ultraviolet B; VZV: Varicella zoster virus; Zn-T8: Zinc transporter 8 


\section{Acknowledgements}

The workshop on managing toxicities associated with immune checkpoint inhibitors was made possible by sponsorship from AstraZeneca Pharmaceuticals, LP; Bristol-Myers Squibb, and Merck \& Co, Inc. Contributions to the content of this white paper from representatives from these companies represent the clinical expertise of the individual authors and not the position of the corporation. Medical writing support for the development of this white paper was provided by Esther Berkowitz at the Society for Immunotherapy of Cancer; no funding support was provided for this purpose.

** The following individuals were contributing authors for the Society for Immunotherapy of Cancer Toxicity Management Working Group: Jeff Anderson, Bristol-Myers Squibb Company, New York, NY. Deborah Arrindell, Amgen, Inc., Thousand Oaks, CA.

Stephanie Andrews, H. Lee Moffitt Cancer Center \& Research Institute, Tampa, FL. Joan Ballesteros, Vivia Biotech S.L., Tres Cantos, Spain

Janie Boyer, AstraZeneca, Gaithersburg, MD.

Daniel Chen, Genentech, Inc., San Francisco, CA.

David Chonzi, Kite Pharma, Los Angeles, CA.

Ion Cotarla, AstraZeneca, Gaithersburg, MD.

Renato Cunha, National Cancer Institute, Bethesda, MD.

Marianne Davies, Yale Cancer Center, New Haven, CT.

Michelle Dawson, AstraZeneca, Gaithersburg, MD*

Adam Dicker, Thomas Jefferson University, Philadelphia, PA.

Lisa Eifler, Prometheus Therapeutics \& Diagnostics, San Diego, CA.

Andrew Ferguson, Gritstone Oncology, Inc., Emeryville, CA.

Cristiano Ferlini, F. Hoffmann La Roche Ltd., New York, NY.

Stanley Frankel, Celgene Corporation, Summit, NJ.

William Go, Kite Pharma, Los Angeles, CA.

Celestine Gochett, KentuckyOne Health, Louisville, KY

Jenna Goldberg, Janssen Pharmaceuticals, Inc., New York, NY.

Priscila Goncalves, HIV and AIDS Malignancy Branch, National Cancer

Institute, National Institutes of Health, Bethesda, MD.

Trishna Goswami, AstraZeneca, Gaithersburg, MD.

Nancy Gregory, Prometheus Therapeutics \& Diagnostics, San Diego, CA.

James L. Gulley, National Cancer Institute, Bethesda, MD.

Vinny Hayreh, AstraZeneca, Gaithersburg, MD.

Nicole Helie, Johns Hopkins Department of Neurology and Neurosurgery

Clinical Trials, Baltimore, MD.

William Holmes, AstraZeneca, Gaithersburg, MD.

Jer-Yuan Hsu, NGM Biopharmaceuticals, Inc., San Francisco, CA.

Ramy Ibrahim, Parker Institute for Cancer Immunotherapy, San Francisco, CA.

Cecilia Larocca, Dana Farber Cancer Institute/Brigham and Women's Hospital, Boston, MA.

Kimberly Lehman, Bristol-Myers Squibb Company, New York, NY.

Sergio Ley-Acosta, Genentech, Inc., San Francisco, CA.

Olivier Lambotte, Assistance Publique Hôpitaux de Paris, France.

Jason Luke, University of Chicago, Chicago, IL.

Joan McClure, National Comprehensive Cancer Network, Fort Washington, PA.

Elisabete Michelon, Pfizer Inc., New York, NY.

Mary Nakamura, University of California, San Francisco, CA.

Kiran Patel, Janssen Pharmaceuticals, Inc., New York, NY.

Bilal Piperdi, Merck \& Co., Kenilworth, NJ.

Zeshaan Rasheed, AstraZeneca, Gaithersburg, MD.

Dan Reshef, Bristol-Myers Squibb Company, New York, NY.

Joanne Riemer, Johns Hopkins Hospital, Baltimore, MD.

Caroline Robert, Institut Gustave Roussy, Villejuif, France.

Makan Sarkeshik, AstraZeneca, Gaithersburg, MD.

Ann Saylors, Bristol-Myers Squibb Company, New York, NY.

Judy Schreiber, AstraZeneca, Gaithersburg, MD.

Kim Shafer-Weaver, AstraZeneca, Gaithersburg, MD.

William Sharfman, Johns Hopkins Medicine, Baltimore, MD.

Elad Sharon, Cancer Therapy Evaluation Program, National Cancer Institute, Bethesda, MD.

Richard Sherry, Surgery Branch, Center for Cancer Research, National Cancer Institute, Bethesda, MD.

Cyndy Simonson, (Un)Common Sense Solutions, Raleigh, NC.

Cherry Thomas, Jounce Therapeutics, Inc., Cambridge, MA.

John A. Thompson, University of Washington/Fred Hutchinson Cancer

Research Center, Seattle, WA.

Elizabeth Trehu, Jounce Therapeutics, Inc., Cambridge, MA.

Dina Tresnan, Pfizer Inc., Groton, CT.
Michelle Turner, Bristol-Myers Squibb Company, New York, NY. Darshan Wariabharaj, Janssen Pharmaceuticals, Inc., New York, NY. Ian Waxman, Bristol-Myers Squibb Company, New York, NY. Lauren Wood, National Cancer Institute, Bethesda, MD.

Lin Zhang, EMD Serono, Inc., Rockland, MA.

Pan Zheng, Children's National Medical Center, Washington D.C.

- Michelle Dawson participated in this workshop while employed by Bristol

Myers Squibb Company, New York, NY, but has since moved to AstraZeneca, Gaithersburg, MD.

\section{Authors' contributions}

IP, AD, KA, COB, CB, RD, LH, SK, MEL, NRL, DL, CO, VS, RS, AWS, DS, MESA, YW, KW, HLK, MSE participated in the drafting of these guidelines and contributed to the writing of the manuscript as well asread and approved the final manuscript. IP, HLK, and MSE organized and lead the workshop where the guidelines were developed.

\section{Authors information}

Dr. Abdallah participated in this workshop while employed by AstraZeneca, Gaithersburg, MD but has since moved to Merck \& Co., Inc.

Dr. Lenihan participated in this workshop while employed at Vanderbilt University Medical Center, Nashville, TN, but has since moved to Washington University in St Louis, St Louis, MO.

\section{Competing interests}

AF declared that he is an employee of Gritstone Oncology, Inc. BP declared that he is an employee and shareholder of Merck \& Co., Inc. CS declared that she is a member of Genentech, Inc. Tecentriq Speakers Bureau. CR declared that she received personal fees through service on the advisory boards at Bristol-Myers Squibb Co., GlaxoSmithKline Pharmaceuticals Ltd., Novartis International AG, Amgen, Inc., Merck \& Co., Inc., and F. Hoffmann-La Roche, Ltd. DC declared that he was employed by Genentech, Inc., until June 2016. DJL declared that he has received personal compensation as a consultant for F. Hoffmann-La Roche, Ltd., Bristol-Myers Squibb Co., and Amgen, Inc., and research support from Takeda Pharmaceuticals U.S.A., Inc. DT declared that she is an employee and shareholder of Pfizer, Inc. DW declared that he has been an employee and shareholder of Janssen Research \& Development, LLC, from May 2016, to present, and an employee of Bayer Healthcare Pharmaceuticals, Inc., from June 2012, to May 2016. IP declared that he received consulting fees from Amgen, Inc., and F. Hoffmann-La Roche, Ltd. JR declared that she is a research nurse at Johns Hopkins University and works with patients receiving $\mathrm{IO}$ agents from multiple sponsors and investigator-initiated studies; JR has also participated in Institute for Clinical Immuno-Oncology Immunotherapy Workshops, has participated in reviewing educational materials developed by AstraZeneca committee and an advisory board meeting sponsored by Merck EMD. JS declared that she is an employee of AstraZeneca Pharmaceuticals. KA declared that he was an employee of AstraZeneca Pharmaceuticals until May 2015, is currently an employee and shareholder of Merck \& Co., Inc., and is a shareholder of Sanofi. KS-W declared that she is an employee of AstraZeneca Pharmaceuticals. MD declared that she has a financial relationship with Speakers Bureau: AstraZeneca Pharmaceuticals; Genentech, Inc.; Merck \& Co., Inc.; Bristol-Myers Squibb Co. ML declared that he has received personal fees from Quintiles Inc., Janssen Research \& Development, LLC, AstraZeneca Pharmaceuticals, Genentech, Inc., Foamix Pharmaceuticals Inc. Infinity Pharmaceuticals, Adgero Biopharmaceuticals Holdings, Inc., Bristol-Myers Squibb Co., and Berg LLC. MS declared that he is an employee of AstraZeneca Pharmaceuticals. MS-A declared that she participated in the Bristol-Myers Squibb Co Advisory Board. OL declared that he has financial relationships with BristolMyers Squibb Co, MSD (Merck \& Co., Inc.) and Janssen Research \& Development, LLC. RD Declared that she receives personal fees from serving on the advisory board at Bristol-Myers Squibb Co. SA declared that she has a financial relationship with Genentech, Inc. Speaker's Bureau. SL declared that he was an employee of Janssen Research \& Development, LLC until January 2016, and is currently an employee of Genentech, Inc. VH declared that she is an employee of AstraZeneca Pharmaceuticals. WS declared that he received consulting fees and general funding from Merck \& Co., Inc., Bristol-Myers Squibb Co, and Novartis International AG. ZR declared that he is an employee of AstraZeneca Pharmaceuticals. All remaining authors declared no competing interests. 


\section{Author details}

Roswell Park Cancer Institute, Elm \& Carlton Streets, Buffalo, NY 14263, USA. ${ }^{2}$ University of Texas MD Anderson Cancer Center, Houston, TX, USA. ${ }^{3}$ Merck \& Co., Inc., Upper Gwynedd, PA, USA. ${ }^{4}$ Johns Hopkins University, Baltimore, MD, USA. ${ }^{5}$ Bristol-Myers Squibb Company, New York, NY, USA. ${ }^{6}$ Memorial Sloan Kettering Cancer Center, New York, NY, USA. 'Dana Farber/Brigham and Women's Cancer Center, Boston, MA, USA. ${ }^{8}$ Washington University in St Louis, St Louis, MO, USA. ${ }^{9}$ Indiana University, Indianapolis, IN, USA.

${ }^{10}$ University of Chicago, Chicago, IL, USA. ${ }^{11}$ Oncology Nursing Society, Pittsburgh, PA, USA. ${ }^{12}$ Massachusetts General Hospital, Boston, MA, USA.

\section{Received: 26 September 2017 Accepted: 6 November 2017} Published online: 21 November 2017

\section{References}

1. Hoos A. Development of immuno-oncology drugs - from CTLA4 to PD1 to the next generations. Nat Rev Drug Discov. 2016;15(4):235-47.

2. Papaioannou NE, et al. Harnessing the immune system to improve cancer therapy. Ann Transl Med. 2016;4(14):261.

3. Administration, U.S.F.a.D. Drugs@FDA: FDA approved drug products. 2017 June 19th, 2017]; Available from: https:/www.accessdata.fda.gov/scripts/ cder/daf/index.cfm.

4. Company, B.-M.S. Ipilimumab (Yervoy) Highlights of Prescribing Information. 2017 3/2017 [cited 2017 June 19th, 2017]; Available from: https:/www. accessdata.fda.gov/drugsatfda_docs/label/2015/125377s073lbl.pdf.

5. Company, B.-M.S. Nivolumab (Opdivo) Highlights of Prescribing Information. 2017 4/2017 [cited 2017 June 19th, 2017]; Available from: https:// packageinserts.bms.com/pi/pi_opdivo.pdf.

6. Merck \& Co., I. Pembrolizumab (Keytruda) Highlights of Prescribing Information. 2017 5/2017 June 19th, 2017]; Available from: https://www. merck.com/product/usa/pi_circulars/k/keytruda/keytruda_pi.pdf.

7. Genentech, I. Atezolizumab (Tecentriq) Highlights of Prescribing Information. 2017 4/2017 [cited 2017 June 19th, 2017]; Available from: https://www.gene.com/download/pdf/tecentriq_prescribing.pdf.

8. LP, A.P. Durvalumab (Imfinzi) Highlights of Prescribing Information. 2017 4/ 2017 [cited 2017 June 19th, 2017]; Available from: https://www.accessdata. fda.gov/drugsatfda_docs/label/2017/761069s000lbl.pdf.

9. EMD Serono, I. Avelumab (Bavencio) Highlights of Prescribing Information. 2017 5/2017 [cited 2017 June 19th, 2017]; Available from: https://www. bavencio.com/en_US/document/Prescribing-Information.pdf.

10. Eggermont $\mathrm{AM}$, et al. Prolonged survival in stage III melanoma with Ipilimumab adjuvant therapy. N Engl J Med. 2016;375(19):1845-55.

11. Bertrand A, et al. Immune related adverse events associated with anti-CTLA4 antibodies: systematic review and meta-analysis. BMC Med. 2015;13:211.

12. Maughan BL, et al. Incidence of immune-related adverse events with program death Receptor-1- and program death Receptor-1 ligand-directed therapies in genitourinary cancers. Front Oncol. 2017;7:56.

13. Villadolid J, Amin A. Immune checkpoint inhibitors in clinical practice: update on management of immune-related toxicities. Transl Lung Cancer Res. 2015;4(5):560-75.

14. Topalian SL, et al. Safety, activity, and immune correlates of anti-PD-1 antibody in cancer. N Engl J Med. 2012;366(26):2443-54.

15. Kumar V, et al. Current diagnosis and Management of Immune Related Adverse Events (irAEs) induced by immune checkpoint inhibitor therapy. Front Pharmacol. 2017;8:49.

16. Ascierto $P A$, et al. Ipilimumab $10 \mathrm{mg} / \mathrm{kg}$ versus ipilimumab $3 \mathrm{mg} / \mathrm{kg}$ in patients with unresectable or metastatic melanoma: a randomised, double-blind, multicentre, phase 3 trial. Lancet Oncol. 2017;18(5):611-22.

17. Tarhini, A.A., et al., A phase III randomized study of adjuvant ipilimumab (3 or $10 \mathrm{mg} / \mathrm{kg}$ ) versus high-dose interferon alfa-2b for resected high-risk melanoma (U.S. Intergroup E1609): Preliminary safety and efficacy of the ipilimumab arms. Journal of Clinical Oncology, 2017. 35(15_suppl): p. 9500-9500.

18. Lin Z, et al. PD-1 antibody monotherapy for malignant melanoma: a systematic review and meta-analysis. PLoS One. 2016;11(8):e0160485.

19. Collins LK, et al. Cutaneous adverse effects of the immune checkpoint inhibitors. Curr Probl Cancer. 2017;41(2):125-8.

20. Hua C, et al. Association of Vitiligo with Tumor Response in patients with metastatic melanoma treated with Pembrolizumab. JAMA Dermatol. 2016;152(1):45-51.

21. Prior, L.M., et al., Toxicities in immunotherapy: Can they predict response? J Clin Oncol, 2016. 34(15_suppl): p. e14534-e14534.

22. Johnson DB, et al. Fulminant myocarditis with combination immune checkpoint blockade. N Engl J Med. 2016;375(18):1749-55.
23. Kwon ED, et al. Ipilimumab versus placebo after radiotherapy in patients with metastatic castration-resistant prostate cancer that had progressed after docetaxel chemotherapy (CA184-043): a multicentre, randomised, double-blind, phase 3 trial. Lancet Oncol. 2014;15(7):700-12.

24. Hodi FS, et al. Improved survival with ipilimumab in patients with metastatic melanoma. N Engl J Med. 2010;363(8):711-23.

25. Ernstoff, M., et al., Challenges faced when identifying patients for combination immunotherapy. Future Oncology, 2017:153(11):1162-1165.

26. Pennock GK, Chow LQ. The evolving role of immune checkpoint inhibitors in cancer treatment. Oncologist. 2015;20(7):812-22.

27. Luke JJ, Ott PA. PD-1 pathway inhibitors: the next generation of immunotherapy for advanced melanoma. Oncotarget. 2015;6(6):3479-92.

28. Spain L, Diem S, Larkin J. Management of toxicities of immune checkpoint inhibitors. Cancer Treat Rev. 2016;44:51-60.

29. Derosa L R.B., Mezquita L, Naltet C, Enot D, Fidelle M, Goubet A-G, Soria JC, Massard C, Goldwasser F, Zitvogel L, Brosseau S, Escudier BJ, Loriot Y, Zalcman G, Besse B, Albiges L., Antibiotics prescription to decrease progression-free survival (PFS) and overall survival (OS) in patients with advanced cancers treated with PD1/PDL1 immune checkpoint inhibitors. J Clin Oncol. 2017;35(Supplement 6S; abstract 462).

30. Kaderbhai $\mathrm{C}$, et al. Antibiotic use does not appear to influence response to Nivolumab. Anticancer Res. 2017;37(6):3195-200.

31. Weber JS, et al. Safety profile of Nivolumab monotherapy: a pooled analysis of patients with advanced melanoma. J Clin Oncol. 2017;35(7):785-92.

32. Horvat TZ, et al. Immune-related adverse events, need for systemic immunosuppression, and effects on survival and time to treatment failure in patients with melanoma treated with Ipilimumab at memorial Sloan Kettering cancer center. J Clin Oncol. 2015:33(28):3193-8.

33. SERVICES., U.S.D.O.H.A.H., Common Terminology Criteria for Adverse Events (CTCAE) Version 4.03. 2010. https://evs.nci.nih.gov/ftp1/CTCAE/CTCAE_4.03_ 2010-06-14_QuickReference_8.5x11.pdf.

34. Woodworth T, et al. Standardizing assessment and reporting of adverse effects in rheumatology clinical trials II: the rheumatology common toxicity criteria v.2.0. J Rheumatol. 2007:34(6):1401-14.

35. Immunotherapy, F.o.C.R.P.I.f.C. Streamlined Toxicity Management Guide_ Working Draft_322.2017. 2017.

36. Belum VR, et al. Characterisation and management of dermatologic adverse events to agents targeting the PD-1 receptor. Eur J Cancer. 2016:60:12-25.

37. Rivera $\mathrm{N}$, et al. Hair Repigmentation during immunotherapy treatment with an anti-programmed cell death 1 and anti-programmed cell death ligand 1 agent for lung cancer. JAMA Dermatol. 2017;153(11):1162-1165.

38. Weber JS, Kähler KC, Hauschild A. Management of Immune-Related Adverse Events and Kinetics of response with Ipilimumab. J Clin Oncol. 2012;30(21):2691-7.

39. Weber JS. Practical management of immune-related adverse events from immune checkpoint protein antibodies for the oncologist. Am Soc Clin Oncol Educ Book. 2012:174-7. https:/meetinglibrary.asco.org/record/66896/edbook.

40. Larkin J, et al. Combined Nivolumab and Ipilimumab or monotherapy in untreated melanoma. N Engl J Med. 2015;373(1):23-34.

41. Weinstock C, et al. U.S. Food and Drug Administration approval summary: Atezolizumab for metastatic non-small cell lung cancer. Clin Cancer Res. 2017; 23(16):4534-453

42. Cramer P, Bresalier RS. Gastrointestinal and hepatic complications of immune checkpoint inhibitors. Curr Gastroenterol Rep. 2017:19(1):3.

43. Oble DA, et al. Alpha-CTLA-4 mAb-associated panenteritis: a histologic and immunohistochemical analysis. Am J Surg Pathol. 2008:32(8):1130-7.

44. Gupta A, et al. Systematic review: colitis associated with anti-CTLA-4 therapy. Aliment Pharmacol Ther. 2015:42(4):406-17.

45. Berman D, et al. Blockade of cytotoxic T-lymphocyte antigen-4 by ipilimumab results in dysregulation of gastrointestinal immunity in patients with advanced melanoma. Cancer Immun. 2010;10:11.

46. Chen JH, et al. Histopathologic features of colitis due to immunotherapy with anti-PD-1 antibodies. Am J Surg Pathol. 2017;41(5):643-54.

47. Weber J, et al. A randomized, double-blind, placebo-controlled, phase II study comparing the tolerability and efficacy of ipilimumab administered with or without prophylactic budesonide in patients with unresectable stage III or IV melanoma. Clin Cancer Res. 2009:15(17):5591-8.

48. Pompili $\mathrm{M}$, et al. Tumor necrosis factor-alpha inhibitors and chronic hepatitis $C$ : a comprehensive literature review. World J Gastroenterol. 2013:19(44):7867-73.

49. Lin MV, et al. The influence of anti-TNF therapy on the course of chronic hepatitis C virus infection in patients with inflammatory bowel disease. Dig Dis Sci. 2013;58(4):1149-56. 
50. Kim KW, et al. Ipilimumab-associated colitis: CT findings. AJR Am J Roentgenol. 2013;200(5):W468-74.

51. Jain A, et al. Colonic ulcerations may predict steroid-refractory course in patients with ipilimumab-mediated enterocolitis. World J Gastroenterol. 2017;23(11):2023-8.

52. Gonzalez RS, et al. PD-1 inhibitor gastroenterocolitis: case series and appraisal of 'immunomodulatory gastroenterocolitis'. Histopathology. 2017;70(4):558-67.

53. Kim KW, et al. Ipilimumab associated hepatitis: imaging and clinicopathologic findings. Investig New Drugs. 2013;31(4):1071-7.

54. Johncilla $M$, et al. Ipilimumab-associated hepatitis: Clinicopathologic characterization in a series of 11 cases. Am J Surg Pathol. 2015;39(8):1075-84.

55. Everett J, Srivastava A, Misdraji J. Fibrin ring granulomas in checkpoint inhibitor-induced hepatitis. Am J Surg Pathol. 2017;41(1):134-7.

56. Marthey L, et al. Cancer immunotherapy with anti-CTLA-4 monoclonal antibodies induces an inflammatory bowel disease. J Crohns Colitis. 2016;10(4):395-401.

57. Ryder $\mathrm{M}$, et al. Endocrine-related adverse events following ipilimumab in patients with advanced melanoma: a comprehensive retrospective review from a single institution. Endocr Relat Cancer. 2014;21(2):371-81.

58. Weber JS, et al. Toxicities of immunotherapy for the practitioner. J Clin Oncol. 2015;33(18):2092-9.

59. Dadu R, Zobniw C, Diab A. Managing adverse events with immune checkpoint agents. Cancer J. 2016;22(2):121-9.

60. Faje A. Immunotherapy and hypophysitis: clinical presentation, treatment, and biologic insights. Pituitary. 2016;19(1):82-92.

61. Marie Joelle Pitteloud RD, Cabanillas ME, Shah K, Mimi I-Nan H, Habra MA, Waguespack SG, et al. Hypophysitis in the Age of Cancer Immunotherapy: Experience in a Large Cancer Center, in Endocrine Society's 97th Annual Meeting and Expo. San Diego: CA; 2015

62. Corsello SM, et al. Endocrine side effects induced by immune checkpoint inhibitors. J Clin Endocrinol Metab. 2013;98(4):1361-75.

63. Blansfield JA, et al. Cytotoxic T-lymphocyte-associated antigen-4 blockage can induce autoimmune hypophysitis in patients with metastatic melanoma and renal cancer. J Immunother. 2005;28(6):593-8.

64. Byun DJ, et al. Cancer immunotherapy - immune checkpoint blockade and associated endocrinopathies. Nat Rev Endocrinol. 2017;13(4):195-207.

65. Johnson DB, et al. Survivorship in immune therapy: assessing chronic immune toxicities, health outcomes, and functional status among long-term Ipilimumab survivors at a single referral center. Cancer Immunol Res. 2015;3(5):464-9.

66. Weber JS, et al. Management of Adverse Events Following Treatment with Anti-Programmed Death-1 agents. Oncologist. 2016;21(10):1230-40.

67. Chamberlain JJ, et al. Diagnosis and Management of Diabetes: synopsis of the 2016 American Diabetes Association standards of medical Care in Diabetes. Ann Intern Med. 2016;164(8):542-52.

68. Naidoo J, et al. Pneumonitis in patients treated with anti-programmed Death-1/programmed death ligand 1 therapy. J Clin Oncol. 2017;35(7):709-17.

69. Khunger $M$, et al. Incidence of pneumonitis with use of PD-1 and PD-L1 inhibitors in non-small cell lung cancer: a systematic review and metaanalysis of trials. Chest. 2017;152(2):271-281.

70. Wu J, et al. PD-1 inhibitors increase the incidence and risk of pneumonitis in cancer patients in a dose-independent manner: a meta-analysis. Sci Rep. 2017;7:44173.

71. Nishino $M$, et al. Incidence of programmed cell death 1 inhibitor-related pneumonitis in patients with advanced cancer: a systematic review and meta-analysis. JAMA Oncol. 2016;2(12):1607-16.

72. Delaunay $\mathrm{M}$, et al. Immune-checkpoint inhibitors associated with interstitial lung disease in cancer patients. Eur Respir J. 2017;50(2). doi:10.1183/ 13993003.00050-2017.

73. Lomax AJ, et al. Immunotherapy-induced sarcoidosis in patients with melanoma treated with PD-1 checkpoint inhibitors: case series and immunophenotypic analysis. Int J Rheum Dis. 2017;20(9):1277-1285.

74. Vogel WV, et al. Ipilimumab-induced sarcoidosis in a patient with metastatic melanoma undergoing complete remission. J Clin Oncol. 2012;30(2):e7-e10.

75. Andersen $\mathrm{R}$, et al. Late development of splenic sarcoidosis-like lesions in a patient with metastatic melanoma and long-lasting clinical response to ipilimumab. Oncoimmunology. 2014;3(8):e954506.

76. Suarez-Almazor ME, et al. Review: immune-related adverse events with use of checkpoint inhibitors for immunotherapy of cancer. Arthritis Rheumatol. 2017:69(4):687-99.

77. Cappelli LC, et al. Rheumatic and musculoskeletal immune-related adverse events due to immune checkpoint inhibitors: a systematic review of the literature. Arthritis Care Res (Hoboken). 2016;69(11):1751-1763.
78. Naidoo J, et al. Inflammatory arthritis: a newly recognized adverse event of immune checkpoint blockade. Oncologist. 2017;22(6):627-30.

79. Weber JS, et al. Safety, efficacy, and biomarkers of nivolumab with vaccine in ipilimumab-refractory or -naive melanoma. J Clin Oncol. 2013;31(34):4311-8.

80. Postow M W.J. Toxicities associated with checkpoint inhibitor immunotherapy. UpToDate 2017 [cited 2017 June 22nd, 2017]; available from: https://www.uptodate.com/contents/toxicities-associated-withcheckpoint-inhibitor-immunotherapy.

81. Gibney GT, et al. Safety, correlative markers, and clinical results of adjuvant nivolumab in combination with vaccine in resected high-risk metastatic melanoma. Clin Cancer Res. 2015;21(4):712-20.

82. Momtaz $\mathrm{P}$, et al. Safety of infusing Ipilimumab over 30 minutes. J Clin Oncol. 2015:33(30):3454-8

83. Bird BR, Swain SM. Cardiac toxicity in breast cancer survivors: review of potential cardiac problems. Clin Cancer Res. 2008;14(1):14-24.

84. Lurz $P$, et al. Diagnostic performance of CMR imaging compared with EMB in patients with suspected myocarditis. JACC Cardiovasc Imaging. 2012;5(5):513-24.

85. Heinzerling L, et al. Cardiotoxicity associated with CTLA4 and PD1 blocking immunotherapy. J Immunother Cancer. 2016:4:50.

86. Shiuan $\mathrm{E}$, et al. Thrombocytopenia in patients with melanoma receiving immune checkpoint inhibitor therapy. J Immunother Cancer. 2017;5:8.

87. Hopkins $A M$, et al. Predicting response and toxicity to immune checkpoint inhibitors using routinely available blood and clinical markers. Br J Cancer. 2017;117(7):913-20.

88. Michot JM, et al. Immune-related adverse events with immune checkpoint blockade: a comprehensive review. Eur J Cancer. 2016:54:139-48.

89. Cortazar FB, et al. Clinicopathological features of acute kidney injury associated with immune checkpoint inhibitors. Kidney Int. 2016;90(3):638-47.

90. Wanchoo R, et al. Adverse renal effects of immune checkpoint inhibitors: a narrative review. Am J Nephrol. 2017;45(2):160-9.

91. Escandon J, et al. Interstitial nephritis in melanoma patients secondary to PD-1 checkpoint inhibitor. J Immunother Cancer. 2017:5:3.

92. Boutros $C$, et al. Safety profiles of anti-CTLA-4 and anti-PD-1 antibodies alone and in combination. Nat Rev Clin Oncol. 2016;13(8):473-86.

93. Rizvi NA, et al. Nivolumab in combination with platinum-based doublet chemotherapy for first-line treatment of advanced non-small-cell lung cancer. J Clin Oncol. 2016:34(25):2969-79.

94. Fadel F, El Karoui K, Knebelmann B. Anti-CTLA4 antibody-induced lupus nephritis. N Engl J Med. 2009;361(2):211-2.

95. Izzedine $\mathrm{H}$, et al. Kidney injuries related to ipilimumab. Investig New Drugs. 2014;32(4):769-73.

96. Thajudeen B, et al. Ipilimumab granulomatous interstitial nephritis. Am J Ther. 2015;22(3):e84-7

97. Kaufman $\mathrm{HL}$, et al. Avelumab in patients with chemotherapy-refractory metastatic Merkel cell carcinoma: a multicentre, single-group, open-label, phase 2 trial. Lancet Oncol. 2016:17(10):1374-85.

98. Cuzzubbo S, et al. Neurological adverse events associated with immune checkpoint inhibitors: review of the literature. Eur J Cancer. 2017:73:1-8.

99. Hottinger AF. Neurologic complications of immune checkpoint inhibitors. Curr Opin Neurol. 2016:29(6):806-12.

100. Bricout M, et al. Vogt-Koyanagi-Harada-like syndrome complicating Pembrolizumab treatment for metastatic melanoma. J Immunother. 2017:40(2):77-82

101. Matsuo T, Yamasaki O. Vogt-Koyanagi-Harada disease-like posterior uveitis in the course of nivolumab (anti-PD-1 antibody), interposed by vemurafenib (BRAF inhibitor), for metastatic cutaneous malignant melanoma. Clin Case Rep. 2017;5(5):694-700

102. Papavasileiou $\mathrm{E}$, et al. Ipilimumab-induced ocular and orbital inflammation-a case series and review of the literature. Ocul Immunol Inflamm. 2016;24(2):140-6.

103. Pages $C$, et al. Ipilimumab-induced acute severe colitis treated by infliximab. Melanoma Res. 2013;23(3):227-30.

104. Tripathi A, et al. Programmed cell death-1 pathway inhibitors in genitourinary malignancies: specific side-effects and their management. Curr Opin Urol. 2016:26(6):548-55 